\title{
Cell wall modifications by $\alpha$-XYLOSIDASE1 are required for the control of seed and fruit size
}

Maurizio Di Marzo ${ }^{1}$, Vívian Ebeling Viana ${ }^{1,2}$, Camilla Banfi ${ }^{1}$, Valeria Cassina ${ }^{3}$, Roberta Corti $^{3,4}$, Humberto Herrera-Ubaldo ${ }^{5}$, Nicola Babolin ${ }^{1}$, Andrea Guazzotti ${ }^{1}$, Edward Kiegle ${ }^{1}$, Veronica Gregis ${ }^{1}$, Stefan de Folter ${ }^{5}$, Javier Sampedro ${ }^{6}$, Francesco Mantegazza ${ }^{3}$, Lucia Colombo ${ }^{1}$, Ignacio Ezquer ${ }^{1}$

1.Dipartimento di BioScienze, Università degli Studi di Milano, Via Giovanni Celoria 26, 20133 Milan, Italy;

2. Plant Genomics and Breeding Center, Federal University of Pelotas, Capão do Leão - RS, Brazil;

${ }^{3}$ School of Medicine and Surgery, Nanomedicine Center NANOMIB, University of Milan-Bicocca, Monza, Italy;

${ }^{4}$ Department of Materials Science, University of Milan-Bicocca, Milan, Italy;

${ }^{5}$ Unidad de Genómica Avanzada (UGA-LANGEBIO), Centro de Investigación y de Estudios Avanzados del Instituto Politécnico Nacional (CINVESTAV-IPN), Km. 9.6 Libramiento Norte, Carretera Irapuato-León, CP 36824 Irapuato, Guanajuato, México;

${ }^{6}$ Universidad de Santiago de Compostela, Departamento de Fisiología Vegetal, Facultad de Biología, Rúa Lope Gómez de Marzoa, s/n. Campus sur, 15782 Santiago de Compostela, A Coruña, Spain.

Corresponding author: Ignacio Ezquer, (juan.ezquer@unimi.it)

(C) The Author(s) 2021. Published by Oxford University Press on behalf of the Society for Experimental Biology. All rights reserved. For permissions, please email: journals.permissions@oup.com 


\section{Highlight}

Cell wall modifications induced by $\alpha$-XYLOSIDASE1, which is regulated by the transcription factor SEEDSTICK, determine seed and fruit size. 


\section{Abstract}

Cell wall modifications are of pivotal importance during plant development. Among cell wall components, xyloglucans are the major hemicellulose polysaccharide in primary cell walls of dicots and non graminaceous monocots. They can connect the cellulose microfibril surface to affect cell wall mechanical properties. Changes in xyloglucan structure are known to play an important role regulating cell growth. Therefore, the degradation of xyloglucan is an important modification that alters the cell wall. The $\alpha$-XYLOSIDASE1 (XYL1) gene encodes the only $\alpha$-xylosidase acting on xyloglucans in Arabidopsis thaliana. Here, we show that mutation of XYL1 strongly influences seed size, seed germination, and fruit elongation. We found that the expression of $X Y L 1$ is directly regulated in developing seeds and fruit by the MADS-box transcription factor SEEDSTICK (STK). We demonstrate that XYL1 complements the stk smaller seed phenotype. Finally, by Atomic Force Microscopy (AFM), we investigate the role of XYL1 activity in maintaining cell stiffness and growth, confirming the importance of cell wall modulation in shaping organs.

\section{Keywords}

Cell wall, fruit growth, MADS-box, seed size, transcription factor, xyloglucan. 


\section{Abbreviations}

ABA - Abscisic acid

ABS - ARABIDOPSIS B SISTER

AFM - Atomic Force Microscopy

AP2 - APETALA 2

ARF - AUXIN RESPONSE FACTOR

BAN - BANYULS/ANTHOCYANIDIN REDUCTASE

bHLH - basic Helix-Loop-Helix

CArG - MADS domain binding sites

CAZY - Carbohydrate active enzymes

CES - CESTA

ChIP - Chromatin immunoprecipitation

CKs - Cytokinin

CKX - CYTOKININ OXIDASE/DEHYDROGENASE

CSLC - CELLULOSE SYNTHASE-LIKE C

CW - Cell wall

DAP - Days after pollination

FUL - FRUITFULL

GA - Gibberellic acid

GH31 - Glycoside hydrolase family 31 
HEC3 - HECATE3

NTT - NO TRANSMITTING TRACT

PME - PECTIN METHYLESTERASE

PMEI - PECTIN METHYLESTERASE INHIBITOR

STK - SEEDSTICK

TF - Transcription factor

WT - Wild-type

XyG - Xyloglucans

XXT1- XYLOGLUCAN XYLOSYLTRANSFERASE 1

XYL1 - $\alpha$-XYLOSIDASE 


\section{Introduction}

The primary plant cell wall (CW) is composed of cellulose microfibrils embedded in a hydrated polysaccharide matrix composed of pectin and hemicelluloses (reviewed in Xiao et al., 2016). Xyloglucans ( $X Y G)$ are the most abundant hemicellulose in the dicot primary CW (reviewed in Pauly and Keegstra, 2016). XyGs are synthesized in the Golgi apparatus by glycan synthases and glycosyltransferases and are delivered to the $\mathrm{CW}$ via vesicular trafficking, where they can undergo structural maturation by apoplastic enzymes (Park and Cosgrove, 2012).

The mechanical properties of the CW are of pivotal importance during development, and it has been shown that XyGs make an important contribution to CW extensibility (Sampedro et al., 2010, 2012; Park and Cosgrove, 2015; Shigeyama et al., 2016). In some XyG-related mutants, like the ones for XYLOGLUCAN XYLOSYLTRANSFERASE1 (XXT1) and XXT2 genes, the levels and patterns of cellulose synthesis are reduced, and this impacts $\mathrm{CW}$ mechanical properties, leading to the reduction of $\mathrm{CW}$ integrity, and rendering the cell sensitive to intrinsic and extrinsic mechanical perturbations (Xiao et al., 2016).

CW remodeling is essential for anisotropic cell expansion (Bashline et al., 2014). The CW of cells in growing, elongating organs must be extensible but at the same time resist the tensile stress ( 10MPa) induced by turgor pressure, which would otherwise irreversibly expand the cell surface area 10 to 1000 -fold (Cosgrove, 2016). The dynamics of the hemicellulose/cellulose crosslinks are thought to be the major factor controlling cell expansion rate, thus regulating plant growth (Iglesias et al., 2006).

During the last years, multidisciplinary tools have been used to uncover the basis of physiological, biochemical, and molecular mechanisms leading to seed germination (Steinbrecher and LeubnerMetzger, 2017). The dynamics of germination has emerged thus as an excellent model system in which an interdisciplinary effort has been made to combine methods from biophysics, engineering, and mathematical sciences providing a sharp focus on the mechanical understanding of plant biology processes.

$\alpha$-XYLOSIDASE1 encoded by XYL1, is classified as one of the carbohydrate-active enzymes (CAZY), specifically a member of the glycoside hydrolase family 31 (GH31) (Sampedro et al., 2001; Cantarel et al., 2009). Loss-of-function of XYL1 results in no detectable $\alpha$-XYLOSIDASE activity (Sampedro et al., 2010). XYL1 has been shown to be important for fruit size determination and anisotropic growth of sepals (Sampedro et al., 2010; Günl and Pauly, 2011; Sechet et al., 2016). Despite the importance of $\alpha$-XYLOSIDASE1 in conferring CW remodeling properties, the molecular control of XYL1 expression 
is still unknown. More recently, viscoelastic properties of $x y / 1$ mutants were examined using creepextension analysis and Force-Indentation experiments to elucidate the alterations of mechanical properties of different tissues like plant stems and flower meristems (Shigeyama et al., 2016; Zhao et al., 2019), confirming that XYL1 function is critical to maintain the rigidity of the primary CW of growing tissues. Since the use of $x y / 1$ mutants is an excellent model to understand nanomechanics in plant systems, in this work, we used a multidisciplinary approach to investigate the network controlling XYL1 expression in seed and fruit size determination.

In recent years, important regulators controlling CW architecture in seed and fruit development have been studied in Arabidopsis thaliana. Thanks to these advances, an extensive and hierarchical genetic model comprising transcription factor (TF) master regulators and their downstream targets acting in CW synthesis and modification have been determined (North et al., 2014; Voiniciuc et al., 2015; Golz et al., 2018; Francoz et al., 2018). We recently demonstrated that the MADS-box TF STK is a fundamental regulator of seed development by virtue of its role in controlling CW properties. Phenotypically, the stk mutant is characterized by smaller seeds and shorter fruits than wild type (WT) (Pinyopich et al., 2003; Herrera-Ubaldo et al., 2019; Di Marzo et al., 2020a). We showed that STK controls pectin modification and cellulose biosynthesis (Ezquer et al., 2016). In addition, stk mutant cannot extrude the pectinaceous mucilage present in the seed coat upon hydration as in a WT plant (Ezquer et al., 2016). The specific control of STK over pectin modification via PECTIN METHYLESTERASE INHIBITOR 6 (PMEI6), influencing seed physiology, is necessary for proper mucilage extrusion and seed germination. However, this transcriptional control over PMEI6 does not affect the control of seed size and fruit size (Ezquer et al., 2016). Nevertheless, it has been reported that STK could impact seed size by regulating the seed coat cell cycle through E2Fa (Paolo et al., 2021). Moreover, STK interacts with NO TRANSMITTING TRACT (NTT) to control genes involved in CW polysaccharide and lipid distribution in the transmitting tract to allow proper fertilization of the ovules (Herrera-Ubaldo et al., 2019). STK also interacts with CESTA (CES), a basic Helix-Loop-Helix (bHLH) TF to control the appropriate CW composition of the septum and consequently of the transmitting tract (Di Marzo et al., 2020b). Recently it has been also demonstrated that the AUXIN RESPONSE FACTOR 6 (ARF6) and ARF8 act with the MADS-box TF FRUITFULL (FUL) to promote fruit elongation via microRNA 172c (miR172c) inhibition of the floral-homeotic gene APETALA 2 (AP2) (José Ripoll et al., 2015) and that STK promotes fruit size determination through the regulation of cytokinin (CK) homeostasis (Di Marzo et al., 2020a).

In this work, we demonstrate that $X Y L 1$ is transcriptionally regulated by STK during fruit and seed development. By mutant analysis, we also investigate the role of XYL1 in conferring CW physical and 
mechanical properties, measuring the CW stiffness using Atomic Force Microscopy-based nanoindentation. This technique has emerged recently as a powerful technique to assess wall stiffness in plant tissues like seeds or floral meristems (Beauzamy et al., 2016; Fourquin et al., 2016; Ezquer et al., 2016; Zhao et al., 2019).

\section{Materials and Methods}

\section{Plant Materials and Growth Conditions}

The $x y l 1$ seed stock's (Col-0 Background, T-DNA insertion line) originate from the GABI-KAT collection (line identifier 749G08). This allele, known as Atxyl1-2, was reported already in Sampedro et al 2010. The $p X Y L 1-G U S$ lines expressed a 3-kb fragment of the XYL1 promoter starting $40 \mathrm{bp}$ from the ATG were provided by J. Sampedro (Sampedro et al., 2010). The Arabidopsis stk mutant seed stocks were provided by M. Yanofsky (Pinyopich et al., 2003). This mutant line contains a 74nucleotide insertion close to the splice site of the third STK intron. Double mutant stk/xyl1 and stk pXYL1-GUS seed stocks were generated by crosses. The pSTK::STK-GFP marker-line used in this work was described in Mizzotti et al., (2014). Seeds were surface sterilized with 70\% ethanol for 2 minutes, $1 \%$ bleach for 5 minutes, then washed three times with sterile water. Seeds were then sown on MS medium (Murashige and Skoog, 1962) and germinated in petri dishes in a growth chamber $\left(22^{\circ} \mathrm{C}\right.$ and 16 hours-light). After 4 days, the seedlings were moved to soil and grown in a greenhouse $\left(22^{\circ} \mathrm{C}\right.$ and 16 hours-light). Mutants were screened using PCR and oligonucleotides (Supplementary Table 1) and consequently phenotyped.

\section{Seed, pistil and silique measurements}

For seed measurements, images were taken with a millimeter scale using a Leica MZ6 stereomicroscope with 5 replicates and 50 seeds each. The images were analyzed using SmartGrain software (Tanabata et al., 2012), to quantify seed size (area size - AS) (refers to the total contents within the border), length (L) (longitudinal length) and width (W) (transverse to longitudinal length). The seed weight measurements were performed with dry seeds in batches of 500 and the weights of at least three replicates were measured for each seed lot and described in text with standard error $( \pm)$. For pistil and silique length and width, photos were taken with a millimeter scale using a stereomicroscope with 5 replicates of 10 pistils and siliques for each genotype analyzed. The pistil 
and silique length and width were analyzed with ImageJ software (Schneider et al., 2012). Statistical analyses were performed using Anova followed by Tukey's, honestly significant difference (HSD) test $(p<0.05, p<0.01)$.

\section{Histochemical analysis}

The pXYL1-GUS marker line was stained for GUS expression to verify the XYL1 activity in ovules, seeds, pistils and siliques of WT compared to stk. Ovule and seed GUS staining was performed as described by Balanzà and co-workers (2016). The samples were analyzed using a Zeiss Axiophot D1 microscope equipped with differential interface contrast (DIC) optics. Images were recorded with an Axiocam MRc5 camera (Zeiss) using the Axiovision program (v.4.1). The pistil and silique XYL1 expression analyses were done as previously described in (Herrera-Ubaldo et al., 2019).

\section{qRT-PCR and Chromatin immunoprecipitation (ChIP) assays}

The RNA was extracted from pistils (stages 10-11-12) and siliques (stage 13-14 and 15-16) collected from the mutant and WT backgrounds. The RNA extracted from seeds (+3 and 4 DAP) was obtained by opening 10 siliques, under stereomicroscope, for each genotype. RNA was obtained using the NucleoSpin RNA, Mini kit for RNA purification (Macherey-Nagel) following the supplier's instructions. Total RNA was retro-transcribed using the iScriptTM gDNA Clear cDNA Synthesis kit (Bio-Rad) following the supplier's instruction. The qRT-PCR assay was done in triplicate using three different biological replicates and three technical replicates within each sample. We used a Bio-Rad iCycler iQ optical system (software version 3.0a). The relative transcript enrichment of XYL1 was calculated by normalizing against two housekeeping genes (ACTIN and UBIQUITIN). Statistical differences were identified with the Student's t test $(* * p<0.01)$.

The genomic region located $3 \mathrm{~kb}$ upstream of the start codon of $X Y L 1$ was analyzed to identify CArG box sequences with up to one base mismatch. ChIP experiments were performed as described in Ezquer et al., (2016). ChIP efficiency was determined using the first CArG box of the VDD gene as a positive control (Matias-Hernandez et al., 2010). Fold enrichment was calculated using ACTIN7 and previously reported formulae (Matias-Hernandez et al., 2010). Sequences of oligonucleotides used for qRT-PCR and ChIP analyses are listed in Supplementary Table 1. CArG box sequences in XYL1 promoter are included in Supplementary Table 2. 


\section{Binary vector construction and Arabidopsis transformation}

For complementation assay the XYL1 locus was cloned from genomic DNA into the pDONR221 entry clone (Invitrogen, Thermo Fisher Scientific, Waltham, MA, USA), and successively into the modified version of $\mathrm{pGWB}, 0$ binary vector containing the STK promoter ( $p S T K:: X Y L 1$ ) and the T35S sequence. Arabidopsis plants were transformed with this construct using the Agrobacterium tumefaciens-mediated floral dip method (Clough and Bent, 1998). Transformant lines were obtained using BASTA as a selection agent. The presence of the construct was assessed by PCR. Sequences of oligonucleotides used are listed in Supplementary Table 1.

\section{AFM}

Atomic force microscopy (AFM) measurements were performed using a Nanowizard II (JPK, Berlin) on single pistils (before fertilization) or fruit at different developmental stages. Dissected valves were physically deposited on the surface of the slide and glued on a double-sided adhesive tape. To prevent significant changes in morphology or biochemistry of living cells, each sample was measured immediately. AFM force spectroscopy consists in imposing a controlled force (ranging from $\mu \mathrm{N}$ to tens of $\mathrm{pN}$ ) to the sample by using a nanometric tip mounted on a micron size cantilever, and in monitoring the deflection of the cantilever, i.e., the applied force, as a consequence of its interaction with the surface (force-distance curves). When the tip is far from the surface there is no cantilever deflection, as there is no interaction between the sample and the tip. As the tip reaches the surface, a force is applied on the sample. As the force value increases, the sample is compressed. The softer the sample, the larger deformation is recorded. At a specific fixed maximum force value (force set point), the cantilever retracts to reach the initial rest position and the pressure is released from the sample surface. The cell stiffness is quantified by extracting the Young modulus (E) proportional to the ratio between the applied force and deformation. The force-displacement curves between contact and 200-nm deformation depth have been analyzed. The evaluation of cell's elastic properties, described quantitatively through the Young Modulus, was obtained by force curves analysis with the Hertz-Sneddon contact mechanics for a paraboloidal tip (Sneddon, 1965; Lekka et al., 1999; Butt et al., 2005). Prior to mechanical experiments, imaging measurements were performed to localize the single cells. Images $\left(50 \times 50 \mu \mathrm{m}^{2}\right.$ or $30 \times 30 \mu \mathrm{m}^{2}, 512 \times 512$ pixels) were acquired in contact mode in water. During that time, force maps (10x10 pixels grid, scan size of $2 \times 2$ $\mu \mathrm{m}^{2}$ ) in the center of the cells were recorded. The force set point was set at $5 \mathrm{nN}$, the approach and retract speeds were kept at $2 \mu \mathrm{m} / \mathrm{s}$ and the length recorded was $2 \mu \mathrm{m}$ in order to have a large portion of the curve recording the non-interaction region between the tip and the sample. The force 
set point chosen allowed a small indentation of the cell, typically less than $200 \mathrm{~nm}$, to ensure to characterize specifically the cell wall properties without any influence by cell turgor. For a given sample type at least 20 cells were measured. Each cantilever (DNP10-C, Nominal Constant $0.24 \mathrm{~N} / \mathrm{m}$, Bruker Corporation) was calibrated before the force measurements both in air and in liquid using the Thermal Noise method (Hutter and Bechhoefer, 1993; Lévy and Maaloum, 2002).

\section{Germination test}

Fresh and non-dormant (stratified) seeds were used to verify the dormancy effects. To overcome dormancy, seeds were stratified at $4^{\circ} \mathrm{C}$ for 7 days (Millar et al., 2006). Seeds of WT (Columbia-0) and mutant plants were grown side-by-side under identical environmental conditions. Seeds were surface sterilized and placed on MS medium (Murashige and Skoog, 1962) without sucrose in a growth chamber $\left(22^{\circ} \mathrm{C}\right.$ and 16 hours-light). Germination was monitored at 24,48 and 72 hours after sowing and the experiment was performed with at least 5 replicates of 30-50 seeds for each mutant and WT. Seeds were scored as germinated when testa rupture was visible. Similar results were obtained with seed stocks from a second set of plants grown independently.

\section{Reciprocal crosses experiments}

Seed measurements and germination \% of seeds obtained from reciprocal crosses were analyzed. The mutants stk and $x y / 1$ were emasculated and then pollinated with WT and/or the respective mutant pollen. The seed measurements were obtained as described above from 3-5 independent biological replicates. Germination \% was scored based on testa rupture of 3-5 independent replicates shown with Standard Deviation (SD).

\section{Mucilage detection and seed abscission analysis}

For mucilage release detection, seeds were stained with $0.01 \%(\mathrm{w} / \mathrm{v})$ ruthenium red (SigmaAldrich) solution for $90 \mathrm{~min}$ under constant agitation. After staining, samples were rinsed in deionized water prior to visualization according to Durand et al., (2009). Seeds were observed and photographed using a stereomicroscope. For seed abscission phenotype we followed the protocol described in Balanzà et al., (2016). 


\section{Xyloglucan analysis}

Approximately $50 \mathrm{mg}$ of a silique pool stages 15 and 16 were homogenized in liquid nitrogen and washed three times with $1 \mathrm{~mL}$ acetone at room temperature. Dried samples were resuspended in $1 \mathrm{~mL}$ of $75 \%$ ethanol for $10 \mathrm{~min}$ and centrifuged at $3000 \mathrm{~g}$ for $5 \mathrm{~min}$. The supernatant, containing free xyloglucan oligosaccharides, was dried. Accessible xyloglucan was extracted from the precipitate, and MALDI-TOF spectra were obtained as previously described (Sampedro et al., 2017). Free oligosaccharides were resuspended in $100 \mu \mathrm{L}$ of water and $2 \mu \mathrm{L}$ of each sample were mixed with $12 \mu \mathrm{L}$ of SDHB matrix and $2 \mu \mathrm{L}$ of $1 \mathrm{mM}$ maltohexaose, to use as an internal standard for quantification. Statistical differences were identified with the Student's t test $(* * p<0.05)$.

Results

\section{SEEDSTICK regulates $X Y L 1$ expression}

Since both mutations, XYL1 and STK, result in a reduction in fruit length (Pinyopich et al., 2003; Günl and Pauly, 2011; Sechet et al., 2016; Shigeyama et al., 2016; Herrera-Ubaldo et al., 2019; Di Marzo et al., 2020a), we questioned whether the smaller seed and shorter silique phenotypes in stk mutant could be linked to a XYL1-dependent mechanism. To study the expression of XYL1, we analyzed the spatio-temporal activity of GUS controlled by a $3 \mathrm{~kb} X Y L 1$ promoter ( $\mathrm{p} X Y L 1$ ). The GUS signal was detected in developing ovules and seeds until globular stage embryo and in developing pistils and siliques from stage 7 to stage 17-B (Fig. 1). Preceding fertilization, $\mathrm{pXYL1}$ activity in stk ovules was similar to WT ovules. The signal was observed in the chalazal region of developing ovules until stage 3-I (according to Schneitz et al., 1995), when the promoter activity is strongest (Fig. 1A). In the subsequent ovule stages the GUS signal drastically decreases (Fig. 1A). After fertilization, the pXYL1 activity is evident throughout the seed starting from 1 day after pollination (1 DAP) until the embryo reaches the globular stage, and then it remains detectable up to the heart stage (Fig. 1A). In the stk mutant the pXYL1 signal was reduced or even absent with respect to WT. This reduction was evident in the seed coat (Fig. 1A).

During WT pistil development, the pXYL1 was active from stage 7 to stage 10 , in the abaxial and adaxial valve of the lateral domain, and in the abaxial margin of the medial domain from stage 10 to 11 (Fig. 1B, C). The pXYL1 at stages 12 and 13 is active in the medial and lateral domain, in replum and valves (Fig. 1B, C, D). At these two stages, in the replum, the pXYL1 activity overlaps with STK 
expression (Herrera-Ubaldo et al., 2019). The GUS expression driven by pXYL1 was lower in stk pistils when compared to WT (Fig. 1B, C, D) and no GUS signal was detected in the replum of stk at stages 12 and 13 (Fig. 1B, C, D). After fertilization, from stages 14 to stage 17-B, the expression of XYL1 and STK overlaps in the replum, where the STK-GFP fusion protein has been already described up to stage 16 (Di Marzo et al., 2020a). However, XYL1 promoter activity increases up to the final stage (17-B) of silique elongation (Fig. 1D). In the stk mutant fruits, the pXYL1 was less active when compared to WT in the valves, while no GUS signal was detected in the replum up to stage 15 . In contrast, a weak signal was detected in the last two stages of fruit development with respect to WT (Fig. 1D), suggesting that STK might be a regulator of $X Y L 1$ expression pattern.

To confirm this hypothesis, we used qRT-PCR to analyze the amount of XYL1 mRNA in WT and stk pistils, siliques and seeds at different developmental stages. The mRNA levels of XYL1 were reduced in stk with respect to WT before fertilization in pistils (stages 10-11-12), during and after fertilization in silique at stages 13-14 and 15-16, but also in seeds at stages 3 and 4 DAP (Fig. 2A). To check whether STK could directly regulate XYL1 transcriptionally, we analyzed the sequence of the promoter region ( $3 \mathrm{~kb}$ upstream of the start codon) of $X Y L 1$ for the presence of MADS domain CArGbox binding sites (Tilly et al., 1998). We identified eight putative CArG-boxes, and we designed primers spanning those genomic regions (Figure 2B and C, see also Supplementary Table 2. We performed a ChIP (chromatin immunoprecipitation) assay using anti-GFP antibodies and developing siliques (up to 6 DAP) of stk mutant plants that expressed pSTK::STK-GFP (Mizzotti et al., 2014). Of the eight CArG-boxes in the XYL1 promoter, the ChIP-qRT assay revealed significant enrichment for binding to the regions spanning CArG-boxes 2 and CArG-boxes 4-5 (Fig. 2B, C). Overall, these observations indicate that STK is a direct regulator of $X Y L 1$.

\section{$X Y L 1$ is required for seed and silique growth}

To characterize STK and XYL1 in different aspects of seed and silique growth, we performed a detailed characterization of mutants disrupted in the genes encoding them. Morphological parameters of seeds collected from stk, $x y / 1$ and double mutant $s t k / x y / 1$ plants were quantified with SmartGrain software (Fig. 3A, B). The $x y / 1$ seeds displayed a bigger seed area than WT and stk, which possesses a smaller seed area when compared to WT (Fig. 3A, B). We found that the increase in length and width of $x y / 1$ seeds resulted in an increase in seed area, while in stk a significant decrease of the seed length caused a reduction of the seed area (Fig. 3A, B). The area of $s t k / x y / 1$ seeds was similar to that of stk without statistically significant differences compared to the single mutant (Fig. 
$3 \mathrm{~A}, \mathrm{~B})$. Seed weight was measured, WT seed weighing an average of $14.8 \pm 0.20 \mathrm{ug} / \mathrm{seed}$ and a significant reduction in stk $13.6 \pm 0.12 \mathrm{ug} / \mathrm{seed}$. The $x y / 1$ seeds possess a similar weight, $15.4 \pm 0.34$ $\mathrm{ug} / \mathrm{seed}$, with respect to WT. Finally, the double mutant seeds $s t k / x y / 1$ weighed an average of $13.5 \pm$ $0.33 \mathrm{ug} / \mathrm{seed}$ similar to stk mutant. The mature ovules of the three mutants displayed no visible phenotypes with respect to WT at anthesis (Supplementary Fig. 1A) demonstrating the role of STK and XYL1 in determining seed size triggered upon fertilization.

Recently it was shown that $\alpha$-XYLOSIDASE1 plays a key role in CW loosening and seed germination, probably controlling a CW integrity signal affecting the regulation of abscisic acid (ABA) and gibberellin (GA) metabolism gene expression in germinating seeds (Shigeyama et al., 2016). To verify the impact of hemicellulose structural alterations, we analyzed in parallel the effects of stk and $x y / 1$ on seed germination. The effect of dormancy on germination was tested using freshly harvested and non-dormant seeds under the same conditions. Germination was observed 24, 48, and $72 \mathrm{~h}$ after sowing both freshly harvested seeds and non-dormant seeds. Interestingly, $x y / 1$ fresh seeds displayed advanced germination (48.6\%) relative to WT (11.2\%) at $24 \mathrm{~h}$ (Table 1). Freshly harvested seeds of stk also displayed earlier germination, reaching $24.5 \%$ germination before $24 \mathrm{~h}$ while nondormant stk seeds germinated in a similar way as the WT ( $43.3 \%$ before $24 \mathrm{~h}$ with respect to $44.6 \%$ for WT). On the other hand, non-additive effects identified in $s t k / x y / 1$ double mutant that displayed a similar germination profile as $x y / 1$. A higher germination rate was also previously observed in freshly harvested seeds of two mutant alleles of XYL1 15 days after sowing (Sechet et al., 2016). Here we demonstrate that the germination potential of $x y / 1$ seeds occurs at the first hours after harvest. Based on these observations, during the early stages of seed germination, $x y / 1$ could be regulated by a yet to be discovered TF since the stk/xyl1 displayed a similar profile observed in $x y / 1$.

It was reported that the anticipated germination upon XYL1 mutation is determined by the endosperm (Sechet et al., 2016). It was recently described also that the stk smaller seed size phenotype is maternally determined (Pinyopich et al., 2003; Paolo et al., 2021). The seed coat is derived from the maternal ovule integuments therefore heterozygous F1 seeds contain zygotic tissues (embryo and endosperm), and this is surrounded by the maternal seed coat. Reciprocal crosses were performed to determine the maternal determinism of altered seed size and germination properties in the $x y / 1$ and stk mutants (Supplementary Fig. 2A, B). Reciprocal crosses did not show differences in the stk and $x y l 1$ seed size, pollinated with WT or the respective mutant pollen indicating the maternal origin of seed size character (Supplementary Fig. 2A).

Germination rates (scored by testa rupture) for freshly harvested seeds showed that F1 seeds, harvested from stk mother plant pollinated with stk pollen, displayed the same phenotype as the F1 
seeds from stk mother crossed with WT pollen (Supplementary Fig. 2B). Thus, the altered germination of stk seeds could result from defective STK in the seed coat layers. Germination rates for F1 seeds harvested from $x y / 1$ mother plants pollinated with WT pollen, displayed the same phenotype as WT (Supplementary Fig. 2B). The $x y / 1$ plants pollinated with mutant pollen produced seeds which displayed a higher percentage of germination with respect to the same mutant pollinated with WT pollen (Supplementary Fig. 2B). This is consistent with experiments reported by Sechet and collaborators (2016) confirming that XYL1 activity in zygotic tissues controls seed germination.

We also investigated whether mutation in $x y l 1$ and double mutation stk/xyl1 can affect seed mucilage extrusion since seed mucilage can positively influence the seed germination process (Western, 2012; Francoz et al., 2015). We observed that $x y / 1$ displayed a WT mucilage extrusion profile based on Ruthenium red staining (Supplementary Fig. 3). Interestingly, stk/xyl1 displayed a $s t k$-mucilage profile but showed a germination rate similar to $x y / 1$. The mucilage phenotypes observed here corroborate with the mucilage role and their hydrogel properties, which enhance seed water uptake during imbibition to regulate seed germination (Zwieniecki et al., 2001).

As mentioned before, STK is a positive regulator of silique size, and like mutants for XYL1, stk mutants develop shorter siliques (Pinyopich et al., 2003; Sampedro et al., 2010; Sechet et al., 2016; Herrera-Ubaldo et al., 2019; Di Marzo et al., 2020a). To compare the morphological effects caused by $x y / 1$ and stk mutation during silique development, mature siliques at stage 17-B from mutant plants were measured with ImageJ software. Both $x y / 1$ and stk displayed shorter siliques than WT, indicating the involvement of these two players in silique development (Fig. 3C, D). These differences between mutants and WT in length were not present at stage 13 when fertilization occurs. In fact, pistils of mutants were similar in length with respect to WT (Supplementary Fig. 1B, C), suggesting that the impact of these mutations occurs specifically during the fruit elongation process. The double mutant $s t k / x y l 1$ displayed a similar silique size pattern as the single mutants (Fig. 3C, D). The stk and $s t k / x y / 1$ lines presented a slightly reduced silique length with respect to the single $x y / 1$ mutant (Fig. 3C, D). Interestingly, the silique shape of the $s t k / x y / 1$ double mutant was similar to the stk single mutant (Fig. 3C, D), although minor statistical differences were present in silique length quantification when compared to stk $(p<0.05)$, confirming that STK acts upstream to $X Y L 1$ to control the silique elongation process. Because $x y / 1$ mutants have been characterized by an increase in fruit width (Sampedro et al., 2010; Shigeyama et al., 2016), we analyzed this phenotype in the single and double mutants (Fig. 3E, F) and also in pistils at stage 13 (Supplementary Fig. 1). The $x y / 1$ mutant exhibited increased fruit width with respect to WT, while the single mutant stk and the 
double mutant stk/xyl1 did not (Fig. 3E, F). The same results were obtained at fertilization where the $x y / 1$ mutant pistils exhibited an increase in width, when compared to WT, while stk and stk/xyl1 possess the same pistil width with respect to WT (Supplementary Fig. 1B, D).

The stk mutant has also been shown to have a defect in seed abscission (Pinyopich et al., 2003; Balanzà et al., 2016). Seed attachment was then analyzed, as stk plants produce seeds well attached to the funiculus (Pinyopich et al., 2003). With the valves opened, $x y / 1$ seeds were dehiscent, just as in WT plants. On the other hand, $x y l 1 / s t k$ seeds were indehiscent, as the stk single mutant (Supplementary Fig. 4). This suggests that seed dehiscence is controlled by STK, probably through a different pathway, which does not involve XYL1.

\section{XYL1 complements the stk smaller seed phenotype}

To investigate whether to what extent the defects in seed size and fruit elongation of stk fruit were due to the role of STK in activating XYL1 expression, we transformed the stk and $x y / 1$ single mutants with a construct expressing the XYL1 locus under the control of the STK promoter (pSTK::XYL1) (Kooiker et al., 2005; Simonini et al., 2012). We generated different lines expressing pSTK::XYL1, and we measured the seed size and silique length in the different genetic backgrounds (Fig. 4 and Supplementary Fig. 5).

We observed that the seed size phenotype upon STK disruption is opposite that upon XYL1 disruption (small seed size phenotype in stk, bigger seed size in $x y / 1$ mutant, compared to WT, Fig. $3 A, B$ respectively). Interestingly, we found that the expression of pSTK::XYL1 in stk allowed the complementation of the seed size differences (area size). In five independent stk transformed lines, seeds expressing PSTK::XYL1 displayed a WT seed size phenotype (Fig. 4A, B). The recovery of the small seed size of stk into an increased WT-like seed size was accompanied by an increase in length and width of the seeds (Fig. 4A, B). We also found that the expression of pSTK::XYL1 in $x y / 1$ permitted the complementation of the seed size. In two independent $x y / 1$ transformed lines, seeds expressing pSTK::XYL1 displayed a decreased and WT-like seed area size phenotype (Fig. 4A, B). The expression of pSTK::XYL1 in WT plants had no seed size effect (Fig. 4A, B).

On the other hand, the silique length in $x y / 1$ expressing pSTK::XYL1, in different independent lines, was not complemented and displayed the same length as the $x y / 1$ mutant (Supplementary Fig. 5). This indicates that the restoration of XYL1 expression in the STK spatiotemporal domain is not sufficient to complement fruit elongation. We also analyzed stk mutant plant lines transformed with 
pSTK::XYL1 and found that the stk short siliques could not be complemented by the insertion of the pSTK::XYL1 construct (Supplementary Fig. 5).

Atomic Force Microscopy (AFM) indentation reveals differences in cell stiffness imposed by STK and $X Y L 1$ during silique elongation.

We investigated whether the altered silique growth of $x y / 1$ and stk single mutants or stk/xyl1 double mutants could translate into a measurable difference in the mechanical properties during the elongation process. Thus we investigated the stiffness of the cells comprising valves from pistils before fertilization (stage 12) and fruit at different developmental stages $(14,15$, and stage 16$)$ by Atomic Force Microscopy (AFM) (Fig. 5).

In unfertilized pistils (stage 12), we found that neither single mutants stk and $x y / 1$ nor double mutant $s t k / x y / 1$ displayed significantly different stiffness compared to WT (Fig. 5). This was also the case post-fertilization, when the fruit elongation process starts (stage 14). However, at stage 15, cell stiffness was significantly increased in $x y / 1$ and in stk/xy/1 double mutants with respect to WT while stk mutants did not differ significantly from WT controls. At stage 16, cell stiffness increased in both $x y / 1$ and stk/xyl1 mutant silique cells with respect to the WT (Fig. 5). stk cell siliques presented a slight increase in stiffness than WT, but this was not statistically significant. Overall, our observations indicate a significant increase in stiffness as fruit growth proceeds, and more specifically upon XYL1 disruption.

\section{Accumulation of xyloglucan oligosaccharides is altered in elongating siliques}

We examined the xyloglucan composition in the different mutants by analyzing free xyloglucan oligosaccharides (XGOs) and accessible xyloglucan in developing siliques. For this experiment we collected a pool of siliques at stages 15 and 16 in which $x y / 1$ showed an increased stiffness compared to WT (Fig. 6).

Both XXXG and XXLG free oligosaccharides were detected in cell walls of $x y / 1$ siliques, at 2.5 and 4.2 $\mathrm{nmol} \mathrm{g}^{-1}$ fresh weight respectively (Fig. 6A). However, they were not detected in stk or stk/xy/1 siliques. When the composition of the enzyme-accessible xyloglucan from the same samples was analyzed, $x y / 1$ siliques showed an accumulation of XXXG and XXLG subunits, but stk and stk/xyl1 samples showed a similar composition to WT (Fig. 6B). The changes observed in $x y / 1$ mutant agree with previous studies of developing pericarp (Shigeyama et al., 2016). 
The analysis of both free oligosaccharides and accessible xyloglucan (Fig. 6A, B) suggests that the reduction in XYL1 expression in stk mutant is insufficient to block the digestion of XXXG and XXLG oligosaccharides or the accumulation of these subunits in polymeric xyloglucan. On the other hand, the lack of detectable oligosaccharides in $s t k / x y / 1$ double mutant, together with a xyloglucan composition similar to that of WT plants would suggest the presence of an $\alpha$-xylosidase activity.

\section{Discussion}

\section{STK controls XYL1 to drive seed and silique development}

STK is a MADS-box TF, which is an essential regulator of many important processes during plant development. Since the initial report of the TF in 2003, which demonstrated a requirement for STK for normal funicular development and ovule identity, several other roles for STK have been elucidated (Pinyopich et al., 2003). STK was shown to be required for the correct formation of the endothelium (the innermost layer of the seed coat) in concert with another MADS-box domain gene, ARABIDOPSIS B SISTER (ABS) (Mizzotti et al., 2012). In addition, STK controls proanthocyanidin metabolism in the seed coat by controlling expression of BANYULS/ANTHOCYANIDIN REDUCTASE $(B A N)$, a key enzyme in proanthocyanidin biosynthesis (Mizzotti et al., 2014). STK was also reported to be a repressor of lignin deposition in the seed abscission zone via negative regulation of HECATE 3 (HEC3) (Balanzà et al., 2016). This finding is consistent with the phenotype observed here for stk and $s t k / x y / 1$, which showed seeds strongly attached to funiculus (Supplementary Fig. 4).

Recently, STK was reported to interact with NTT and CES to control genes involved in CW polysaccharide and lipid distribution in gynoecium medial domain cells (Herrera-Ubaldo et al., 2019; Di Marzo et al., 2020b). STK was also found to be expressed during fruit development and maturation, showing expression from 3 to 12 DAP (Mizzotti et al., 2018). Lastly, it was recently demonstrated that STK may control degradation of cytokinins by transcriptionally regulating CYTOKININ OXIDASE/DEHYDROGENASE 7 (CKX7), and in so doing, exerts some control over the elongation of the fruit (Di Marzo et al., 2020a). Therefore, another CK-independent mechanism of STK fruit control could act via XYL1.

Since the first report that XYL1 encodes $\alpha$-XYLOSIDASE 20 years ago (Sampedro et al., 2001), numerous research groups have revealed the critical role of hemicellulose in plant development. XYL1 encodes for an apoplastic $\alpha$-XYLOSIDASE able to generate Xyl from xyloglucan oligosaccharides 
(Sampedro et al., 2001). Later, the expression pattern of XYL1 was reported in young and mature leaves, roots, in the apical and basal region of stems, flowers and siliques (Iglesias et al., 2006). Expression of XYL1 was also reported in cell types undergoing CW modifications such as trichomes, vasculature, stomata, and anther filaments (Sampedro et al., 2010).

Guided by some similarities in expression patterns in pistil and fruit structures, we studied in detail the control of STK over XYL1 in ovule/seeds, pistil and fruit tissues. We analyzed pXYL1 activity in stk at specific time points covering those used for expression analysis, using the pXYL1::GUS construct (Fig. 1). The comparative spatio-temporal analysis of $p X Y L 1:: G U S$ pattern revealed a decreased signal in stk mutant with respect to WT, in particular in the seed coat, where STK protein is expressed (Mizzotti et al., 2014) (Fig. 1). The same result was obtained in a detailed characterization in siliques after fertilization. A decrease in GUS signal was detected in particular in replum, where the two genes are co-expressed (Di Marzo et al., 2020a and Fig. 1), and in the silique valves where only XYL1 is expressed (Fig. 1). The quantification of XYL1 mRNA levels confirmed that XYL1 expression was lower in stk than in WT (Fig. 2). We also showed that STK is a direct regulator of XYL1 by demonstrating that STK can bind directly to specific regions of the XYL1 promoter (regions spanning CArG-boxes 2 and 4-5) (Fig. 2). These observations may form the basis for future studies to determine the impact or importance of each STK binding site on XYL gene expression. In this regard, it would be interesting to use partial (or combined) mutations of these XYL promoter sites to analyze how STK is deregulated in different reproductive tissues. Interestingly, to investigate whether the defect in seed size or fruit length in stk was due to the low levels of $X Y L 1$, we generated transgenic lines on stk mutants expressing the XYL1 locus under the control of the STK promoter. As shown in Fig. 4, the stk seed size was restored, indicating that the restoration of XYL1 expression in the stk mutant can complement the seed size.

\section{Lack of XYL1 affects seed and silique growth}

In this work we have characterized at the seed and fruit level $x y / 1$, stk, and stk/xyl1 mutants. The $x y / 1$ mutant displayed a bigger seed area with respect to WT caused by an increase in the width of that seeds (Fig. 3). A decrease of fruit length in $x y / 1$ mutants was described previously, along with increased silique width caused by wider pericarp cells (Sampedro et al., 2010; Günl and Pauly, 2011; Sechet et al., 2016). We observed that stk mutants also have smaller seeds accompanied by a decrease in seed length (Fig. 3). The impact in the organ area by alterations in elongation processes could work in a similar way as it occurs in the siliques of stk mutant. In fact, the smaller stk fruit is a 
consequence of the lack of cell valve elongation (Di Marzo et al., 2020a). We found that the $s t k / x y / 1$ double mutant does not display an additive effect, and the double mutant phenotype resembled the stk single mutant (Fig. 3).

In agreement with previously published work, we observed an increased pistil and silique width for the single mutant $x y / 1$. However, the pistil and silique width of double mutant $s t k / x y / 1$ and the single mutant stk resembled that of WT (Fig. 3 and Supplementary Fig. 1).

The introgression of PSTK::XYL1 construct into stk and $x y / 1$ allowed complementation of the altered seed size but did not allow the complementation of the stk and $x y / 1$ silique size (Fig. 4 and Supplementary Fig. 5).

These observations indicate that the control of STK in seed size is fully dependent on the control of the expression of XYL1. These results also suggest that the proper balance between STK-XYL1 levels is determinant to maintain the proper seed growth.

Regarding fruit growth, it seems that the control of STK in silique elongation is not dependent on the control of the expression of $X Y L 1$, and other factors are involved in the STK-dependent fruit elongation process. It has been shown that STK influences elongation via multiple pathways like controlling the expression of the FUL-AP2 pathway and through the regulation of CK concentrations (Di Marzo et al., 2020a). Therefore, one hypothesis is that these two pathways predominate with respect to the STK-XYL1 pathway.

XYL1 disruption influences the mechanical properties and xyloglucan composition of elongating siliques

We demonstrate here that STK is critical for fine control of $X Y L 1$ expression in fruits during elongation process (Fig. 1). This could influence the mechanical properties of the fruit CW during elongation. To assess this, we used AFM to check how stk and $x y / 1$ mutations can affect the nanomechanical properties of growing siliques (Fig. 5). We have detected the cell stiffness on valves cells because it has been demonstrated that increased fruit size results from the elongation of these cells (Vivian-Smith et al., 2001). Silique elongation, from fertilized pistils up to the final fruit size, is a process separated in a first brief phase in which cell division occurs and a subsequent step in which cell expansion and elongation predominate (Vivian-Smith and Koltunow, 1999). 
We measured a significant increase in stiffness for the single mutant $x y / 1$ and the double mutant stk/xyl1 at stage 15 forward, compared to stk and WT (Fig. 5). However, at stage 16, double mutant $s t k / x y / 1$ siliques showed similar stiffness to stk while $x y / 1$ showed an increased stiffness compared to WT and its stiffness at stage 15 (Fig. 5). These data suggest that differences in cell stiffness during the silique elongation process depend on xyloglucan alterations.

The composition analysis of the free xyloglucan oligosaccharides and accessible polymeric xyloglucan in developing siliques revealed differences between $x y l 1$ and stk siliques. We detected an accumulation of XXXG and XXLG subunits in elongating $x y / 1$ siliques, but stk siliques possessed a similar composition to WT (Fig. 6). We hypothesize that although the expression of XYL1 in stk is reduced but not null (Fig. 2), this may account for the normal xylosidase activity required to maintain normal xyloglucan homeostasis as reported by Monroe and collaborators (2003). The partial compensation of the $x y / 1$ mechanical cell stiffness in the double mutant stk/xyl1 again confirms that STK acts upstream of XYL1 to regulate CW alterations and consequently cell stiffness, which is essential for normal silique elongation. Overall, we showed that the CW properties of stiffness and weakness coordinate silique expansion and, therefore, silique growth. Shigeyama and co-workers (2016) reported that silique epidermal cells upon disruption of XYL1 are longitudinally shorter and horizontally enlarged, and that the elasticity and viscosity (obtained by creep-extension analyses) of the $\mathrm{CW}$ in the elongating part of flower stem mutants were lower than WT. These findings, together with our data, support the role of XYL1 in controlling silique properties via CW stiffness. The AFM results presented here could be compared to the study of the viscoelastic properties reported in the literature on analogous mutants by creep-extension analysis or tensile testing (Tanimoto et al., 2000; Shigeyama et al., 2016). The decrease of elasticity in the $x y l 1$ mutant measured by creep-extension analysis by Shigeyama and co-workers agree with our data showing an increase in stiffness measured by AFM force spectroscopy in developing siliques (Fig. 5), even if the two techniques differ in working principles, methods, and scale ( $\mathrm{mm}$ vs. hundreds of $\mathrm{nm}$ ). Namely, indentation techniques such as AFM give local mechanical information with high spatial and force resolution. At the same time, tensile testing provides measurements on the whole sample, which is stretched along one or two directions in order to extract its bulk elastic, viscoelastic, and plastic properties (Yakubov et al., 2016). Furthermore, creep-extensions analysis measures the in-plane tensile mechanical properties, while AFM involves other mechanical details such as material deformation of the CW thickness (Milani et al., 2011). For example, it has been suggested that "in-plane" tensile mechanics is sensitive to the properties of the cellulose microfibril network, while out-of-plane indentation mechanics depends on the bendability and elasticity of the deformed cellulose and homogalacturonan networks (Zhang et al., 2019; Robinson and Durand-Smet, 2020). Overall, the 
measured indentation Young modulus values are generally lower with respect to the values measured in tension (Bidhendi and Geitmann, 2016), and given the heterogeneous structure under investigation, the relation of tensile properties of the wall with the indentation measurements is a difficult task (Mosca et al., 2017).

\section{Proposed models}

Because seed size in stk mutant was restored by the localized expression of XYL1 driven by the STK promoter, it follows that the control of hemicellulose homeostasis exerted via XYL1 is a major regulator of seed size control downstream of STK. Recently, it was reported that STK influences seed size via the cell cycle regulator E2Fa in the developing seed coat (Paolo et al., 2021). With this and previous evidence we hereby provide an updated model regarding the STK control of seed growth (Fig. 7A).

Considering the fruit size, we hypothesize that STK acts upstream of, but not exclusively over, XYL1 to control fruit length. This is consistent with our observations using AFM, which demonstrated that mechanically $x y l 1$ mutant behaves in a different way with respect to stk and stk/xyl1. In a similar way, we show that altered XG composition of $x y / 1$ is not observed in the stk and stk/xy/1 genetic backgrounds. The lack of detectable XG oligosaccharides in $s t k / x y / 1$ double mutant (where XYL1 activity is not present) may suggest the presence of an alternative xylosidase activity. Alternatively, to $\mathrm{CW}$ control, STK also acts through a) hormonal homeostasis via cytokinin degradation and b) "non-cell autonomous" regulation of the transcriptional FUL-AP2 pathway to control fruit elongation (Di Marzo et al., 2020a). To prove or disprove this, further experiments should be done in the relevant mutants with altered fruit phenotypes to see if the regulation of xylosidase activity is central to these pathways to control fruit elongation or independent (Fig. 7B).

Comprehensive CW models will aid in the redesign of plant CWs for the purpose of agronomically viable production, aiming to improve fundamental commercial traits such as seed and fruit yield, and fruit maturation processes. Here, we propose the integration of molecular, biochemical, developmental, and biophysical studies on a larger scale to determine the full potential of CWmodifying agents for the modulation of differentiation processes. 


\section{Acknowledgements}

This work was partially supported by the H2020-MSCA- RISE Project (ExpoSeed GA-691109) and European Union FP7 project EVOCODE (grant number 247587), Research Projects of National Interest (PRIN) PRIN201719LCOLO_01. The research was supported by Università degli Studi di Milano (Piano di sviluppo di ateneo Linea 2-DBS Ezquer and Linea 3-SEED). Work in the SDF laboratory is financed by the Mexican National Council of Science and Technology (CONACYT) grant CB-2017-2018-A1-S-10126. We thank L. Ceccato for technical support.

\section{Author contributions}

I.E and LC conceived the original work and research plans, L.C, I. E., S.D, F. M supervised the experiments. M.D, V.E.V, I.E, C.B, N.B, H.H, V.C, V.G performed the experiments, R.C, A.G, V.G provided technical assistance. M.D, V.E.V, J.S, L.C, I.E designed the experiments and analyzed the data. M.D, V.E.V, J.S, I.E and L.C conceived the project and wrote the article with contributions of all the authors, E.K, F.M, S.D supervised and completed the writing. I.E agrees to serve as the author responsible for contact and ensures communication.

\section{Conflicts of interest}

There are no conflicts of interest.

\section{Data availability}

All data supporting the findings of this study are available within the paper and within its supplementary materials published online. 


\section{References}

Balanzà V, Roig-Villanova I, Di Marzo M, Masiero S, Colombo L. 2016. Seed abscission and fruit dehiscence required for seed dispersal rely on similar genetic networks. Development 143, 3372-3381.

Bashline L, Lei L, Li S, Gu Y. 2014. Cell wall, cytoskeleton, and cell expansion in higher plants. Molecular Plant 7, 586-600.

Beauzamy L, Fourquin C, Dubrulle N, Boursiac Y, Boudaoud A, Ingram G. 2016. Endosperm turgor pressure decreases during early Arabidopsis seed development. Development 143, 3295-3299.

Bidhendi AJ, Geitmann A. 2016. Relating the mechanics of the primary plant cell wall to morphogenesis. Journal of Experimental Botany 67, 449-461.

Butt HJ, Cappella B, Kappl M. 2005. Force measurements with the atomic force microscope: Technique, interpretation and applications. Surface Science Reports 59, 47-57.

Cantarel BI, Coutinho PM, Rancurel C, Bernard T, Lombard V, Henrissat B. 2009. The CarbohydrateActive EnZymes database (CAZy): An expert resource for glycogenomics. Nucleic Acids Research 37, D233-D238.

Clough SJ, Bent AF. 1998. Floral dip: A simplified method for Agrobacterium-mediated transformation of Arabidopsis thaliana. Plant Journal 16, 735-743.

Cosgrove DJ. 2016. Catalysts of plant cell wall loosening. F1000Research 5, 119.

Di Marzo M, Herrera-Ubaldo H, Caporali E, Novák O, Strnad M, Balanzà V, Ezquer I, Mendes MA, de Folter S, Colombo L. 2020a. SEEDSTICK Controls Arabidopsis Fruit Size by Regulating Cytokinin Levels and FRUITFULL. Cell Reports 30, 2846-2857.e3.

Di Marzo M, Roig-Villanova I, Zanchetti E, et al. 2020b. MADS-box and bHLH transcription factors coordinate transmitting tract development in arabidopsis thaliana. Frontiers in Plant Science 11, 1-16.

Durand C, Vicré-Gibouin M, Follet-Gueye ML, Duponchel L, Moreau M, Lerouge P, Driouich A. 2009. The organization pattern of root border-like cells of Arabidopsis is dependent on cell wall homogalacturonan. Plant Physiology 150, 1411-1421.

Ezquer I, Mizzotti C, Nguema-Ona E, et al. 2016. The developmental regulator SEEDSTICK controls structural and mechanical properties of the arabidopsis seed coat. Plant Cell 28, 2478-2492.

Fourquin C, Beauzamy L, Chamot S, Creff A, Goodrich J, Boudaoud A, Ingram G. 2016. Mechanical stress mediated by both endosperm softening and embryo growth underlies endosperm elimination in Arabidopsis seeds. Development 143, 3300-3305.

Francoz E, Lepiniec L, North HM. 2018. Seed coats as an alternative molecular factory: thinking outside 
the box. Plant Reproduction 31, 327-342.

Francoz E, Ranocha P, Nguyen-Kim H, Jamet E, Burlat V, Dunand C. 2015. Roles of cell wall peroxidases in plant development. Phytochemistry 112, 15-21.

Golz JF, Allen PJ, Li SF, Parish RW, Jayawardana NU, Bacic A, Doblin MS. 2018. Layers of regulation Insights into the role of transcription factors controlling mucilage production in the Arabidopsis seed coat. Plant Science 272, 179-192.

Günl M, Pauly M. 2011. AXY3 encodes a $\alpha$-xylosidase that impacts the structure and accessibility of the hemicellulose xyloglucan in Arabidopsis plant cell walls. Planta 233, 707-719.

Herrera-Ubaldo H, Lozano-sotomayor P, Ezquer I, et al. 2019. New roles of NO TRANSMITTING TRACT and SEEDSTICK during medial domain development in arabidopsis fruits. Development 146, dev172395.

Hutter JL, Bechhoefer J. 1993. Manipulation of van der Waals forces to improve image resolution in atomic-force microscopy. Journal of Applied Physics 73, 4123-4129.

Iglesias N, Abelenda JA, Rodiño M, Sampedro J, Revilla G, Zarra I. 2006. Apoplastic glycosidases active against xyloglucan oligosaccharides of Arabidopsis thaliana. Plant and Cell Physiology 47, 55-63.

José Ripoll J, Bailey L, Mai QA, Wu SL, Hon CT, Chapman EJ, Ditta GS, Estelle M, Yanofsky MF. 2015. MicroRNA regulation of fruit growth. Nature Plants 1, 1-9.

Kooiker M, Airoldi CA, Losa A, Manzotti PS, Finzi L, Kater MM, Colombo L. 2005. BASIC PENTACYSTEINE1, a GA Binding Protein That Induces Conformational Changes in the Regulatory Region of the Homeotic Arabidopsis Gene SEEDSTICK. The Plant Cell 17, 722 LP-729.

Lekka M, Laidler P, Gil D, Lekki J, Stachura Z, Hrynkiewicz AZ. 1999. Elasticity of normal and cancerous human bladder cells studied by scanning force microscopy. European Biophysics Journal 28 312-316.

Lévy R, Maaloum M. 2002. Measuring the spring constant of atomic force microscope cantilevers: Thermal fluctuations and other methods. Nanotechnology 13, 33-37.

Matias-Hernandez L, Battaglia R, Galbiati F, Rubes M, Eichenberger C, Grossniklaus U, Kater MM, Colombo L. 2010. VERDANDI is a direct target of the MADS domain ovule identity complex and affects embryo sac differentiation in Arabidopsis. Plant Cell 22, 1702-1715.

Milani P, Gholamirad M, Traas J, Arnéodo A, Boudaoud A, Argoul F, Hamant O. 2011. In vivo analysis of local wall stiffness at the shoot apical meristem in Arabidopsis using atomic force microscopy. Plant Journal 67, 1116-1123.

Millar AA, Jacobsen J V., Ross JJ, Helliwell CA, Poole AT, Scofield G, Reid JB, Gubler F. 2006. Seed dormancy and ABA metabolism in Arabidopsis and barley: The role of ABA 8'-hydroxylase. Plant Journal 45, 942-954.

Mizzotti C, Ezquer I, Paolo D, et al. 2014. SEEDSTICK is a Master Regulator of Development and 
Metabolism in the Arabidopsis Seed Coat. PLoS Genetics 10. e1004856.

Mizzotti C, Mendes MA, Caporali E, Schnittger A, Kater MM, Battaglia R, Colombo L. 2012. The MADS box genes SEEDSTICK and ARABIDOPSIS Bsister play a maternal role in fertilization and seed development. Plant Journal 70, 409-420.

Mizzotti C, Rotasperti L, Moretto M, Tadini L, Resentini F, Galliani BM, Galbiati M, Engelen K, Pesaresi $P$, Masiero S. 2018. Time-course transcriptome analysis of arabidopsis siliques discloses genes essential for fruit development and maturation. Plant Physiology 178, 1249-1268.

Monroe JD, Garcia-Cazarin ML, Poliquin KA, Aivano SK. 2003. Antisense Arabidopsis plants indicate that an apoplastic $\alpha$-xylosidase and $\alpha$-glucosidase are encoded by the same gene. Plant Physiology and Biochemistry 41, 877-885.

Mosca G, Sapala A, Strauss S, Routier-Kierzkowska AL, Smith RS. 2017. On the micro-indentation of plant cells in a tissue context. Physical Biology 14, 015003.

Murashige T, Skoog F. 1962. A Revised Medium for Rapid Growth and Bio Assays with Tobacco Tissue Cultures. Physiologia Plantarum 15, 473-497.

North HM, Berger A, Saez-Aguayo S, Ralet M-CC. 2014. Understanding polysaccharide production and properties using seed coat mutants: Future perspectives for the exploitation of natural variants. Annals of Botany 114, 1251-1263.

Paolo D, Rotasperti L, Schnittger A, Masiero S, Colombo L, Mizzotti C. 2021. The arabidopsis madsdomain transcription factor seedstick controls seed size via direct activation of e2fa. Plants 10, 1-8.

Park YB, Cosgrove DJ. 2012. Changes in cell wall biomechanical properties in the xyloglucan-deficient xxt1/xxt2 mutant of Arabidopsis. Plant Physiology 158, 465-475.

Park YB, Cosgrove DJ. 2015. Xyloglucan and its interactions with other components of the growing cell wall. Plant and Cell Physiology 56, 180-194.

Pauly M, Keegstra K. 2016. Biosynthesis of the Plant Cell Wall Matrix Polysaccharide Xyloglucan. Annual Review of Plant Biology 67, 235-259.

Pinyopich A, Ditta GS, Savidge B, Liljegren SJ, Baumann E, Wisman E, Yanofsky MF. 2003. Assessing the redundancy of MADS-box genes during carpel and ovule development. Nature 424, 85-88.

Robinson S, Durand-Smet P. 2020. Combining tensile testing and microscopy to address a diverse range of questions. Journal of Microscopy 278, 145-153.

Sampedro J, Gianzo C, Iglesias N, Guitián E, Revilla G, Zarra I. 2012. AtBGAL10 Is the Main Xyloglucan $\beta$ Galactosidase in Arabidopsis, and Its Absence Results in Unusual Xyloglucan Subunits and Growth Defects. Plant Physiology 158, 1146-1157.

Sampedro J, Pardo B, Gianzo C, Guitián E, Revilla G, Zarra I. 2010. Lack of $\alpha$-xylosidase activity in 
arabidopsis alters xyloglucan composition and results in growth defects. Plant Physiology 154, 11051115.

Sampedro J, Sieiro C, Revilla G, González-Villa T, Zarra I. 2001. Cloning and expression pattern of a gene encoding an $\alpha$-xylosidase active against xyloglucan oligosaccharides from arabidopsis. Plant Physiology 126, 910-920.

Sampedro J, Valdivia ER, Fraga P, Iglesias N, Revilla G, Zarra I. 2017. Soluble and membrane-bound $\beta$ glucosidases are involved in trimming the Xyloglucan backbone. Plant Physiology 173, 1017-1030.

Schneider CA, Rasband WS, Eliceiri KW. 2012. NIH Image to ImageJ: 25 years of image analysis. Nature Methods 9, 671-675.

Schneitz K, Hülskamp M, Pruitt RE. 1995. Wild-type ovule development in Arabidopsis thaliana: a light microscope study of cleared whole-mount tissue. The Plant Journal 7, 731-749.

Sechet J, Frey A, Effroy-Cuzzi D, et al. 2016. Xyloglucan metabolism differentially impacts the cell wall characteristics of the endosperm and embryo during Arabidopsis seed germination. Plant Physiology 170, 1367-1380.

Shigeyama T, Watanabe A, Tokuchi K, Toh S, Sakurai N, Shibuya N, Kawakami N. 2016. $\alpha$-Xylosidase plays essential roles in xyloglucan remodelling, maintenance of cell wall integrity, and seed germination in Arabidopsis thaliana. Journal of Experimental Botany 67, 5615-5629.

Simonini S, Roig-Villanova I, Gregis V, Colombo B, Colombo L, Kater MM. 2012. BASIC PENTACYSTEINE proteins mediate MADS domain complex binding to the DNA for Tissue-Specific expression of target genes in Arabidopsis. Plant Cell 24, 4163-4172.

Sneddon IN. 1965. The relation between load and penetration in the axisymmetric boussinesq problem for a punch of arbitrary profile. International Journal of Engineering Science 3, 47-57.

Steinbrecher T, Leubner-Metzger G. 2017. The biomechanics of seed germination. Journal of Experimental Botany 68, 765-783.

Tanabata T, Shibaya T, Hori K, Ebana K, Yano M. 2012. SmartGrain: High-throughput phenotyping software for measuring seed shape through image analysis. Plant Physiology 160, 1871-1880.

Tanimoto E, Fujii S, Yamamoto R, Inanaga S. 2000. Measurement of viscoelastic properties of root cell walls affected by low pH in lateral roots of Pisum sativum L. Plant and Soil 226, 21-28.

Tilly JJ, Allen DW, Jack T. 1998. The CArG boxes in the promoter of the Arabidopsis floral organ identity gene APETALA3 mediate diverse regulatory effects. Development 125, 1647-1657.

Vivian-Smith A, Koltunow AM. 1999. Genetic analysis of growth-regulator-induced parthenocarpy in Arabidopsis. Plant Physiology 121, 437-451.

Vivian-Smith A, Luo M, Chaudhury A, Koltunow A. 2001. Fruit development is actively restricted in the 
absence of fertilization in Arabidopsis. Development 128, 2321-2331.

Voiniciuc C, Yang B, Schmidt MHW, Günl M, Usadel B. 2015. Starting to gel: How arabidopsis seed coat epidermal cells produce specialized secondary cell walls. International Journal of Molecular Sciences 16, 3452-3473.

Western TL. 2012. The sticky tale of seed coat mucilages: Production, genetics, and role in seed germination and dispersal. Seed Science Research 22, 1-25.

Xiao C, Zhang T, Zheng Y, Cosgrove DJ, Anderson CT. 2016. Xyloglucan deficiency disrupts microtubule stability and cellulose biosynthesis in arabidopsis, altering cell growth and morphogenesis. Plant Physiology 170, 234-249.

Yakubov GE, Bonilla MR, Chen H, Doblin MS, Bacic A, Gidley MJ, Stokes JR. 2016. Mapping nano-scale mechanical heterogeneity of primary plant cell walls. Journal of Experimental Botany 67, 2799-2816.

Zhang T, Tang H, Vavylonis D, Cosgrove DJ. 2019. Disentangling loosening from softening: insights into primary cell wall structure. Plant Journal 100, 1101-1117.

Zhao F, Chen W, Sechet J, et al. 2019. Xyloglucans and microtubules synergistically maintain meristem geometry and phyllotaxis. Plant Physiology 181, 1191-1206.

Zwieniecki MA, Melcher PJ, Holbrook NM. 2001. Hydrogel Control of Xylem Hydraulic Resistance in Plants. Science 291, 1059-1062. 
Table

Table 1. Germination test of freshly harvested and non-dormant (stratified) seeds of stk, xyl1 and stk/xyl1 mutants compared to WT.

\begin{tabular}{|c|c|c|c|c|c|c|c|c|c|c|c|c|}
\hline \multirow{3}{*}{$\begin{array}{l}\text { Geno } \\
\text { type }\end{array}$} & \multicolumn{6}{|c|}{ Fresh harvested seed } & \multicolumn{4}{|c|}{ Non-dormant seeds } & & \\
\hline & \multicolumn{2}{|c|}{$24 \mathrm{~h}$} & \multicolumn{2}{|c|}{$48 \mathrm{~h}$} & \multicolumn{2}{|c|}{$72 \mathrm{~h}$} & \multicolumn{2}{|c|}{$24 \mathrm{~h}$} & \multicolumn{2}{|c|}{$48 \mathrm{~h}$} & \multicolumn{2}{|c|}{$72 \mathrm{~h}$} \\
\hline & $\%$ & SD & $\%$ & SD & $\%$ & SD & $\%$ & SD & $\%$ & SD & $\%$ & SD \\
\hline Col & 11.2 & 2.3 & 42.8 & 1.6 & 77.4 & 1.5 & 44.6 & 1.5 & 75.2 & 2.2 & 90.8 & 3.4 \\
\hline stk & 24.5 & 1.9 & 77.2 & 3.52 & 91.3 & & 43.3 & 1.4 & 83.8 & 2.7 & 91.5 & 1.8 \\
\hline$x y / 1$ & 48.6 & 2.5 & 87.3 & 4.9 & 0 & 5. & 60.7 & 1.7 & 93.7 & 4.1 & 95.25 & 2.8 \\
\hline stk/xyl1 & 52.8 & 1.93 & 84.5 & 3.2 & 89.9 & 2.45 & 60.3 & 2 & 89.5 & 3.5 & 93.3 & 4.0 \\
\hline
\end{tabular}


Figure Legends

Fig. 1: Detailed spatiotemporal distribution of $X Y L$ promoter activity in stk ovule, seed, pistils and fruits compared to WT.

(A) Stereomicroscope images showing pXYL1::GUS activity in stk and WT ovules, before fertilization, and seed after fertilization from 1 DAP (day after fertilization) to heart stage, ch: chalaza, sc: seed-coat, es: embryo sac, em: embryo; Scale bars: 20 and $50 \mu \mathrm{m}$. (B) Schematic representation and description of the various structures of pistils and fruits at stage 7 and 12 before fertilization, 13 during fertilization process and 17-B when the fruit reaches its maximum size and length; Scale bars: $1 \mathrm{~mm}$. (C) Cross sections showing expression of XXYL1::GUS from stage 7 to 12 in stk and WT pistils; Scale bars: $100 \mu \mathrm{m}$. (D) Cross sections showing expression of $p X Y L 1:: G U S$ from stage 13 to 17-B in stk and WT fruits; Scale bars: $100 \mu \mathrm{m}$.

Fig. 2: STK is a direct regulator of $X Y L 1$.

A) Analysis of XYL1 expression before fertilization/pollination in carpels (stages 10, 11 and 12), in developing pistil and fruit from stage 13 to 16 and in seeds at stage 3-4 DAP in stk mutant compared to WT; Error bars represent the propagated error value of three technical replicate; Statistical analysis was performed using Student's t test (** $p<0.01$ ). (B) Representative ChIP experiment between pSTK::STKGFP and WT. Error bars represent the SD for three technical replicates. The fold enrichment was calculated against data for WT. Binding of STK to a CArG-box in the VERDANDI (VDD, CArG-1) promoter was used as a positive control. (C) Schematic representation of the localisation of the putative CArGboxes in the $3 \mathrm{~kb} X Y L 1$ promoter region (* indicate positive STK-binding regions based on ChIP experiment).

Fig. 3: Seeds and silique phenotypes in stk and $x y / 1$ single mutants and in the double mutant stk/xyl1 compared to WT.

A) Histogram representing seed size of the single and double mutants with respect to WT, with seed area, length and width of the seed; Error bars represent the SD. $n=300$ seeds. Statistical analyses were performed using Anova followed by Tukey's HSD test. Different letters indicate statistically significant differences $(p<0.01)$. Statistical differences among the various genotypes related to seed size are indicated in upper case text; lower case text is used to describe statistical differences related to seed length, and italicized lower case to describe seed width. B) Stereomicroscope images of stk, $x y / 1$ and stk/xyl1 seeds compared to WT; Scale bar: $0.2 \mathrm{~mm}$. C) Histogram representing fruit length of the single and double mutants with respect to WT; Error bars represent the SD. $n=50$ siliques. Statistical analyses 
were performed using Anova followed by Tukey's HSD test. Different letters indicate statistically significant differences $(p<0.01)$.

* Among stk and $s t k / x y l 1$ the statistically significant difference is $p<0.05$. D) Stereomicroscope images of stk, $x y / 1$ and $s t k / x y / 1$ siliques compared to WT at stage 17-B; Scale bar: $1 \mathrm{~mm}$. E) Histogram representing fruit width of the single and double mutants with respect to WT. Error bars represent the SD. $n=50$ siliques. Statistical analyses were performed using Anova followed by Tukey's HSD test. Different letters indicate statistically significant differences $(p<0.01)$. F) Stereomicroscope images of stk, $x y / 1$ and stk/xyl1 siliques compared to WT at stage 17-B with focus on fruit width; Scale bar: $1 \mathrm{~mm}$.

Fig. 4: Seed measurements of complementation experiment.

(A) Histogram representing seed size of the single mutants stk and $x y / 1$ complemented with pSTK::XYL respect to single mutants not transformed with pSTK::XYL and WT, with seed area, length and width of the seed; Error bars represent the SD. $n=300$ seeds. Statistical differences among the various genotypes related to seed size are indicated in upper case text; lower case text is used to describe statistical differences related to seed length and italicized lower case to describe seed width. (B) Stereomicroscope images of the genotypes described above; Scale bar: $0.1 \mathrm{~mm}$. Statistical analyses were performed using Anova followed by Tukey's HSD test. Different letters indicate statistically significant differences $(p<$ 0.01 ) between each mutant and genotype lines with respect to WT.

\section{Fig. 5: AFM analysis on developing fruits.}

(A) Comparison of the apparent stiffness of $s t k, x y / 1$ and $s t k / x y l 1$ mutant fruits compared to WT at different developmental stages (stage 12 before fertilization, stages 14, 15 and 16 after fertilization during fruit elongation process) which are depicted at the bottom with respect to the box plots (scale bar: $1 \mathrm{~mm}$ ). Stiffness values were extracted as Young Modulus values from the fit Hetz model of the force displacement curves in which each value represents the average Young modulus per cell square of over 100 force curves, with the associated SD. Differences between populations were evaluated statistically using a Wilcoxon rank-sum test $(* p<0.05, * * p<0.01)$. (B) Stereomicroscope images of WT pistil and siliques at the stages analyzed in AFM experiment; Scale bar: $1 \mathrm{~mm}$. 
Fig. 6: Xyloglucan characterization in siliques of $s t k, x y l 1$ and $s t k / x y / 1$.

(A) Histogram representing free oligosaccharides extracted from siliques at stage 15-16 of stk, $x y / 1$ and $s t k / x y / 1$ with respect to WT. Error bars represent the SD. $n=5$. (B) Histogram representing the proportion of accessible xyloglucan extracted from the siliques of $s t k, x y / 1$ and $s t k / x y l 1$ with respect to WT. Error bars represent the SD. Statistical differences were identified with Student's t test $\left({ }^{* *} p<\right.$ 0.05). Mutants marked with asterisks were significantly different with respect to WT control or among them if connected.

Fig. 7: Updated models for STK transcriptionally regulating XYL1 in the control of seed and fruit size.

(A) Proposed model for seed growth controlled by STK upstream of XYL1; STK also controls directly E2Fa to regulate seed coat cell cycle (see also (Paolo et al., 2021)). (B) Proposed model for silique growth controlled by STK upstream of XYL1. STK directly controls Cytokinin (CKs) homeostasis activating CKX7 and indirectly FUL which plays a crucial role in the valve elongation (see also (Di Marzo et al., 2020a)). 
A

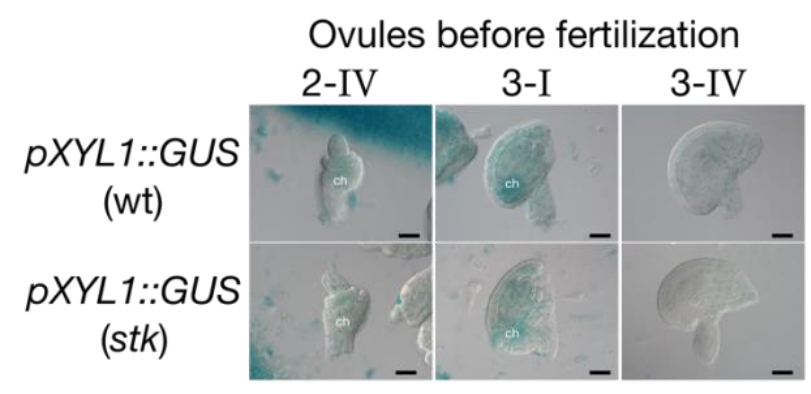

Seed after fertilization

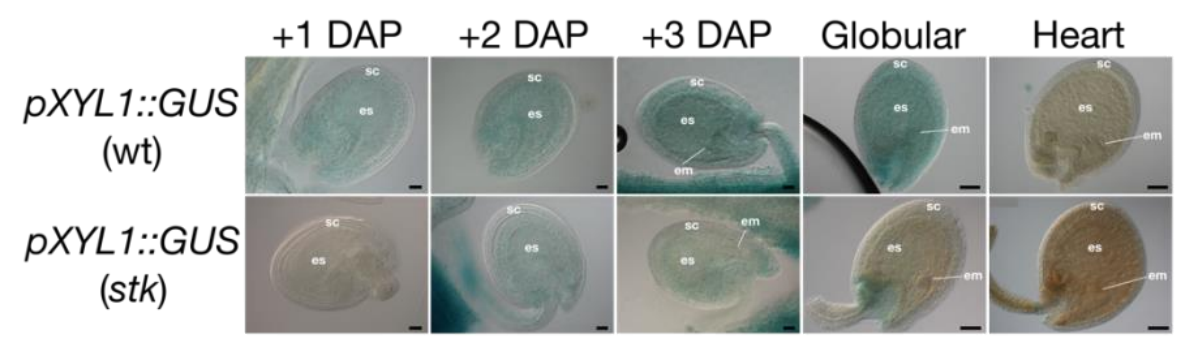

B

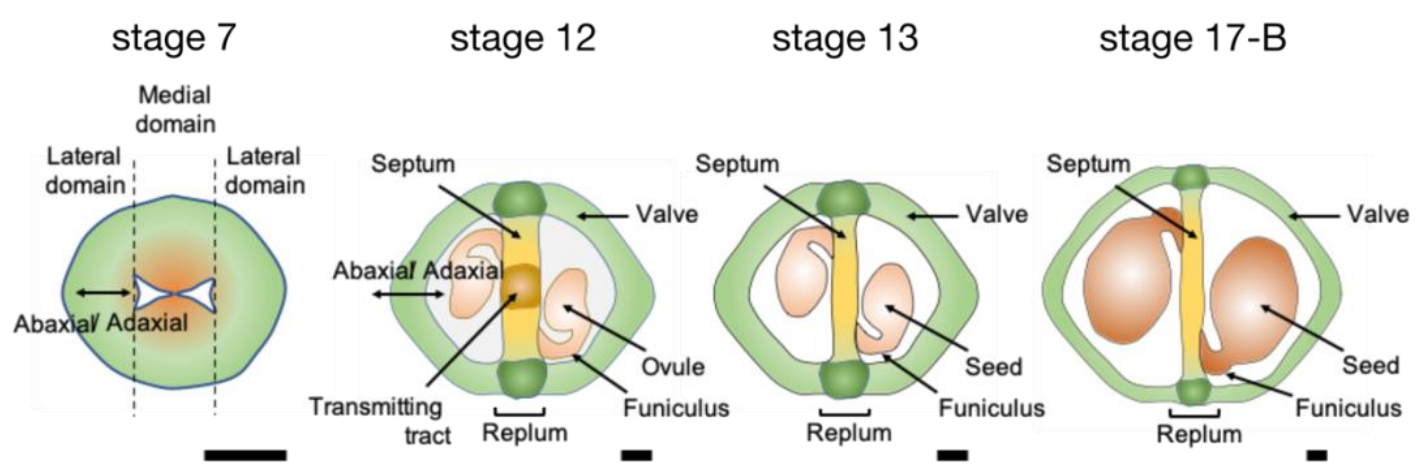

C
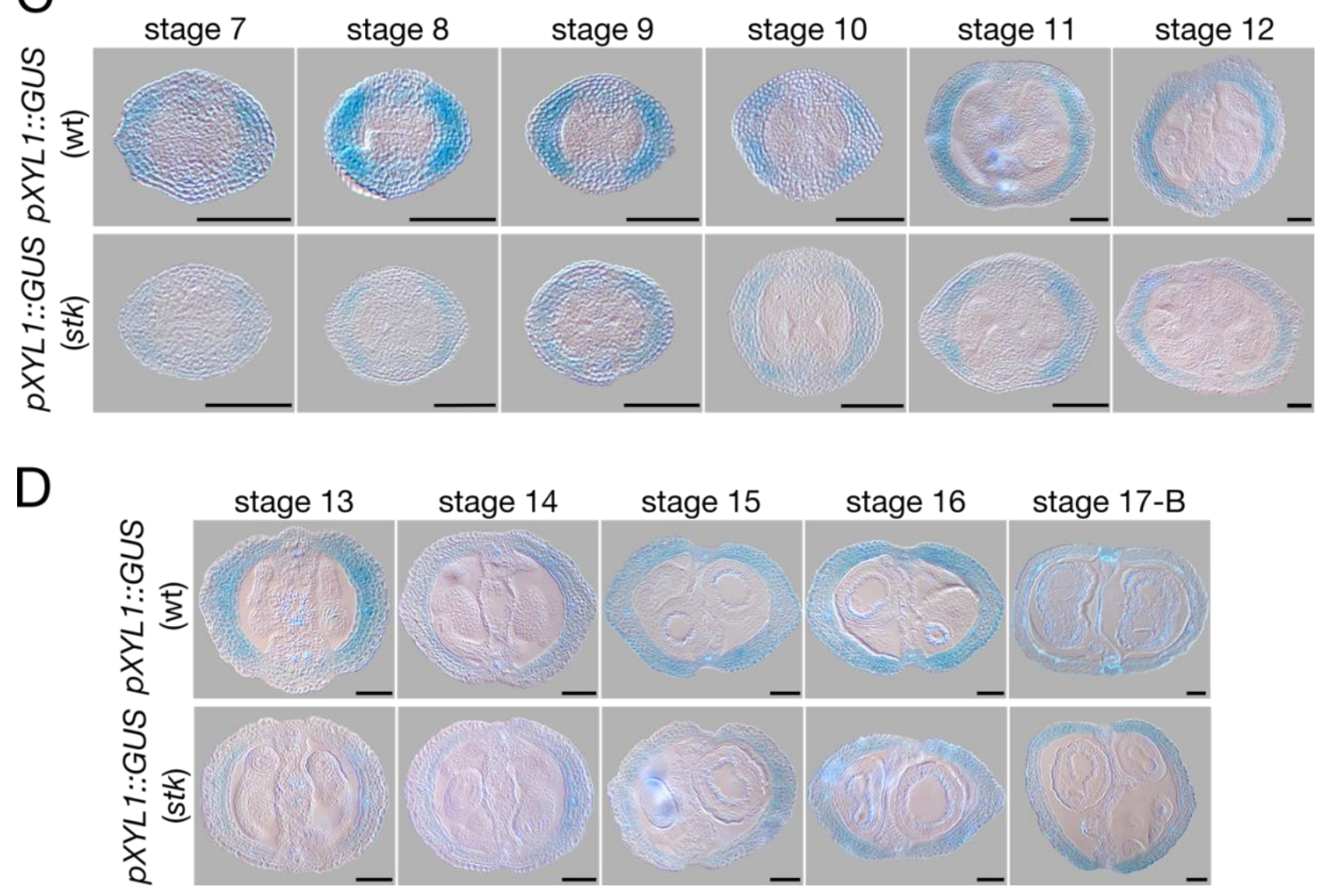
Fig. 1: Detailed spatiotemporal distribution of $X Y L$ promoter activity in stk ovule, seed, pistils and fruits compared to WT.

(A) Stereomicroscope images showing $p X Y L 1:: G U S$ activity in stk and WT ovules, before fertilization, and seed after fertilization from 1 DAP (day after fertilization) to heart stage, ch: chalaza, sc: seed-coat, es: embryo sac, em: embryo; Scale bars: 20 and $50 \mu \mathrm{m}$. (B) Schematic representation and description of the various structures of pistils and fruits at stage 7 and 12 before fertilization, 13 during fertilization process and 17-B when the fruit reaches its maximum size and length; Scale bars: $1 \mathrm{~mm}$. (C) Cross sections showing expression of pXYL1::GUS from stage 7 to 12 in stk and WT pistils; Scale bars: $100 \mu \mathrm{m}$. (D) Cross sections showing expression of $p X Y L 1: \because G U S$ from stage 13 to $17-\mathrm{B}$ in stk and WT fruits; Scale bars: $100 \mu \mathrm{m}$. 
A

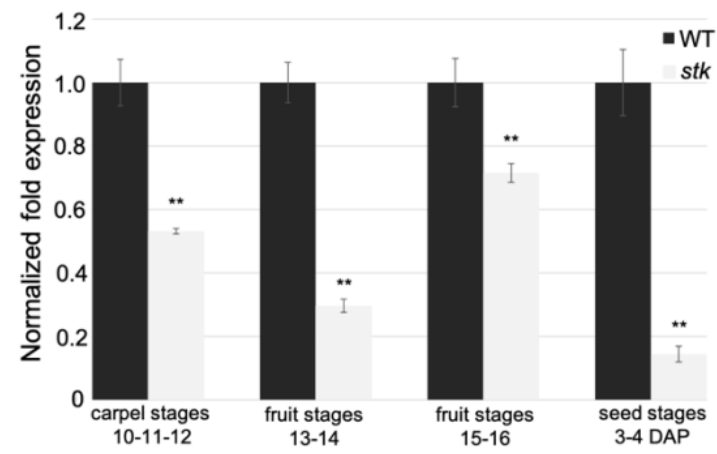

C
B

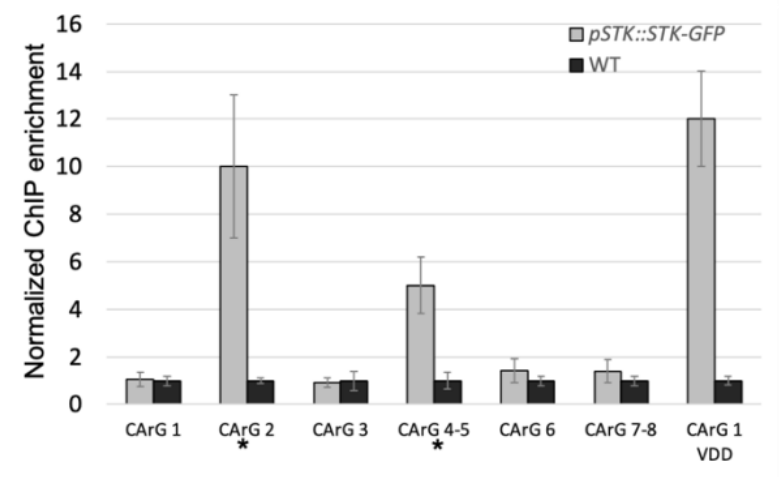

Fig. 2: STK is a direct regulator of $X Y L 1$.

A) Analysis of XYL1 expression before fertilization/pollination in carpels (stages 10, 11 and 12), in developing pistil and fruit from stage 13 to 16 and in seeds at stage 3-4 DAP in stk mutant compared to WT; Error bars represent the propagated error value of three technical replicate; Statistical analysis was performed using Student's t test $\left.*^{* *} \mathrm{p}<0.01\right)$. (B) Representative ChIP experiment between pSTK::STK-GFP and WT. Error bars represent the SD for three technical replicates. The fold enrichment was calculated against data for WT. Binding of STK to a CArG-box in the VERDANDI (VDD, CArG-1) promoter was used as a positive control. (C) Schematic representation of the localisation of the putative CArG-boxes in the $3 \mathrm{~kb}$ XYL1 promoter region (* indicate positive STK-binding regions based on ChIP experiment). 


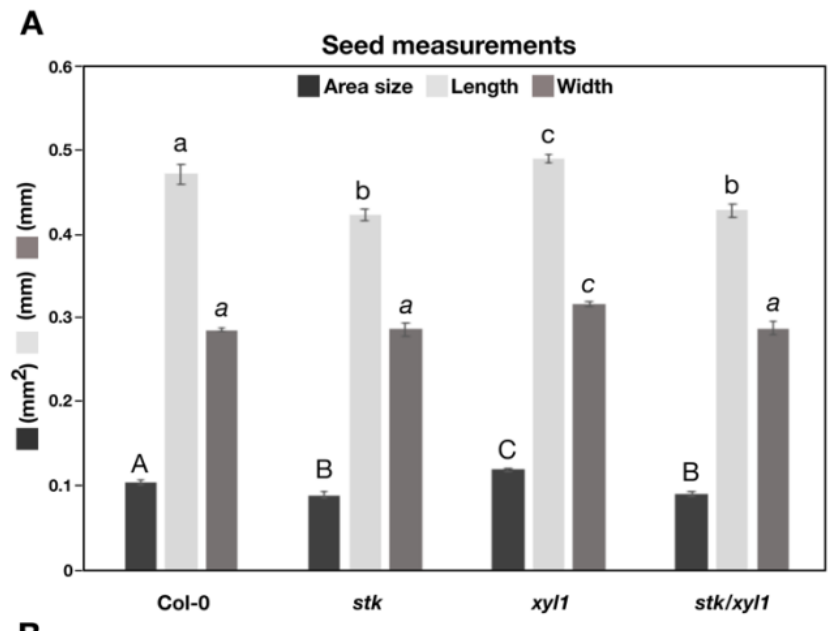

B



C

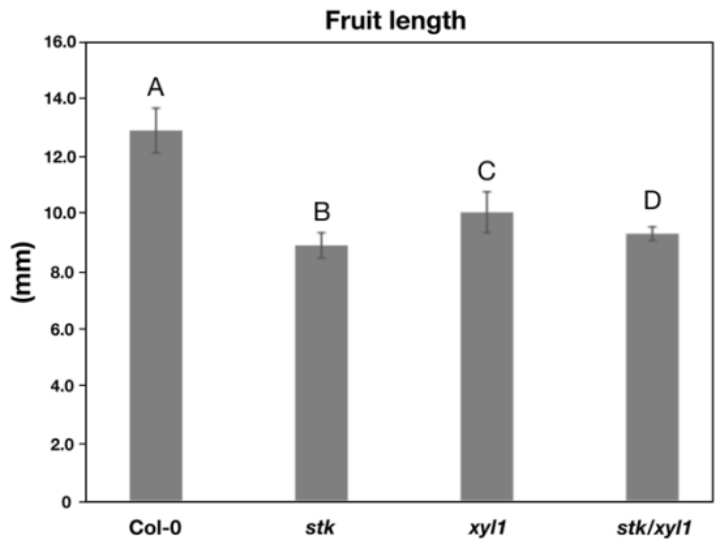

D

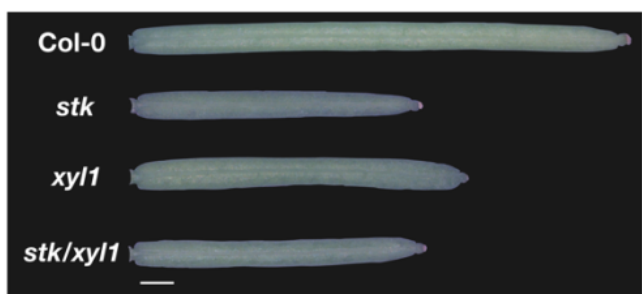

E

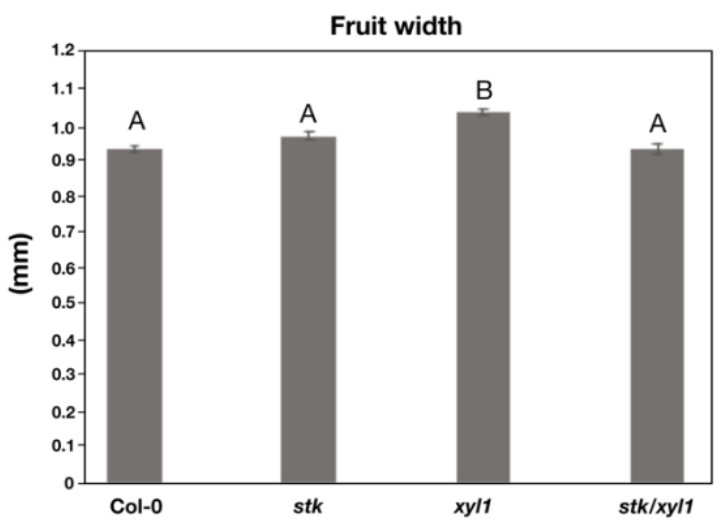

F

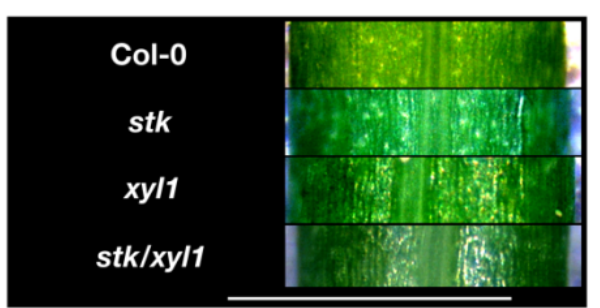


Fig. 3: Seeds and silique phenotypes in stk and xyll single mutants and in the double mutant stk/xyll compared to WT.

A) Histogram representing seed size of the single and double mutants with respect to WT, with seed area, length and width of the seed; Error bars represent the SD. $n=300$ seeds. Statistical analyses were performed using Anova followed by Tukey's HSD test. Different letters indicate statistically significant differences $(\mathrm{p}<0.01)$. Statistical differences among the various genotypes related to seed size are indicated in upper case text; lower case text is used to describe statistical differences related to seed length, and italicized lower case to describe seed width. B) Stereomicroscope images of stk, xyll and stk/xyll seeds compared to WT; Scale bar: $0.2 \mathrm{~mm}$. C) Histogram representing fruit length of the single and double mutants with respect to WT; Error bars represent the SD. $n=50$ siliques. Statistical analyses were performed using Anova followed by Tukey's HSD test. Different letters indicate statistically significant differences $(\mathrm{p}<0.05$ and $\mathrm{p}<0.01)$. D) Stereomicroscope images of stk, xyll and stk/xyll siliques compared to WT at stage 17-B; Scale bar: $1 \mathrm{~mm}$. E) Histogram representing fruit width of the single and double mutants with respect to WT. Error bars represent the SD. $n=50$ siliques. Statistical analyses were performed using Anova followed by Tukey's HSD test. Different letters indicate statistically significant differences $(\mathrm{p}<0.01)$. F) Stereomicroscope images of stk, xyll and stk/xyll siliques compared to WT at stage 17-B with focus on fruit width; Scale bar: $1 \mathrm{~mm}$. 
A

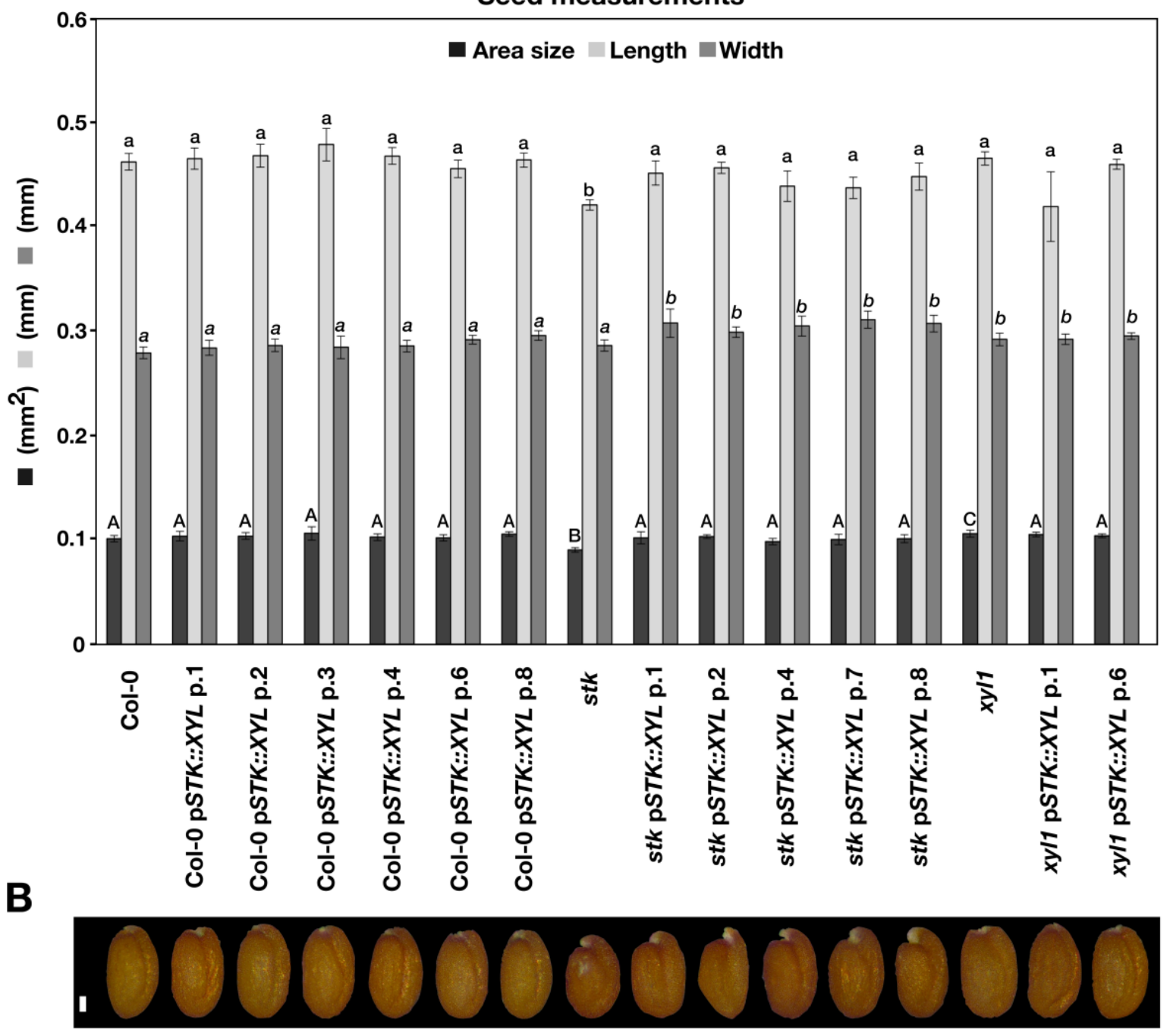

Fig. 4: Seed measurements of complementation experiment.

(A) Histogram representing seed size of the single mutants stk and xyll complemented with $\mathrm{p} S T K:: X Y L$ respect to single mutants not transformed with $\mathrm{p} S T K:: X Y L$ and WT, with seed area, length and width of the seed; Error bars represent the SD. $n=300$ seeds. Statistical differences among the various genotypes related to seed size are indicated in upper case text; lower case text is used to describe statistical differences related to seed length and italicized lower case to describe seed width. (B) Stereomicroscope images of the genotypes described above; Scale bar: $0.1 \mathrm{~mm}$. Statistical analyses were performed using Anova followed by Tukey's HSD test. Different letters indicate statistically significant differences $(p<0.01)$ between each mutant and genotype lines with respect to WT. 


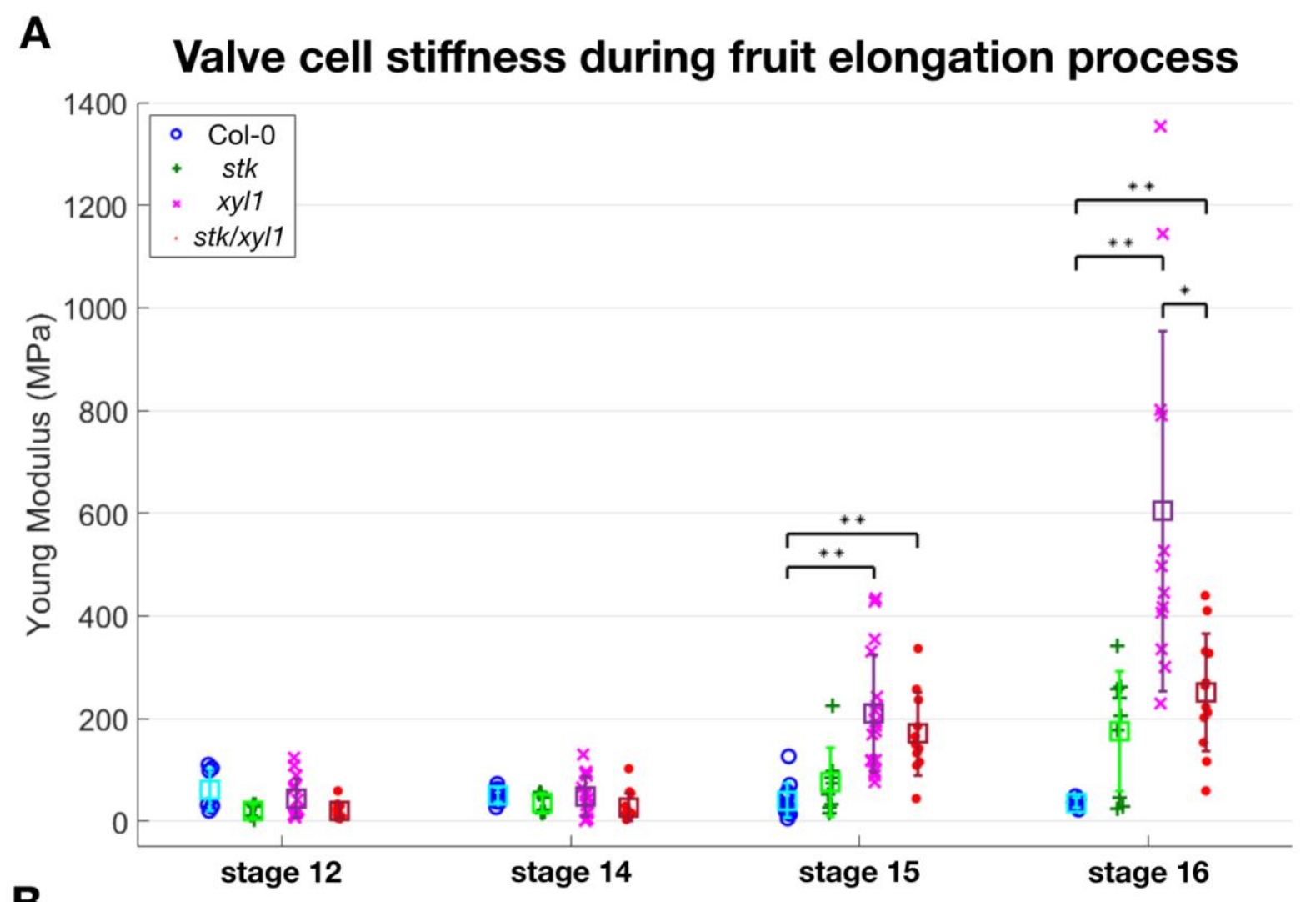

B

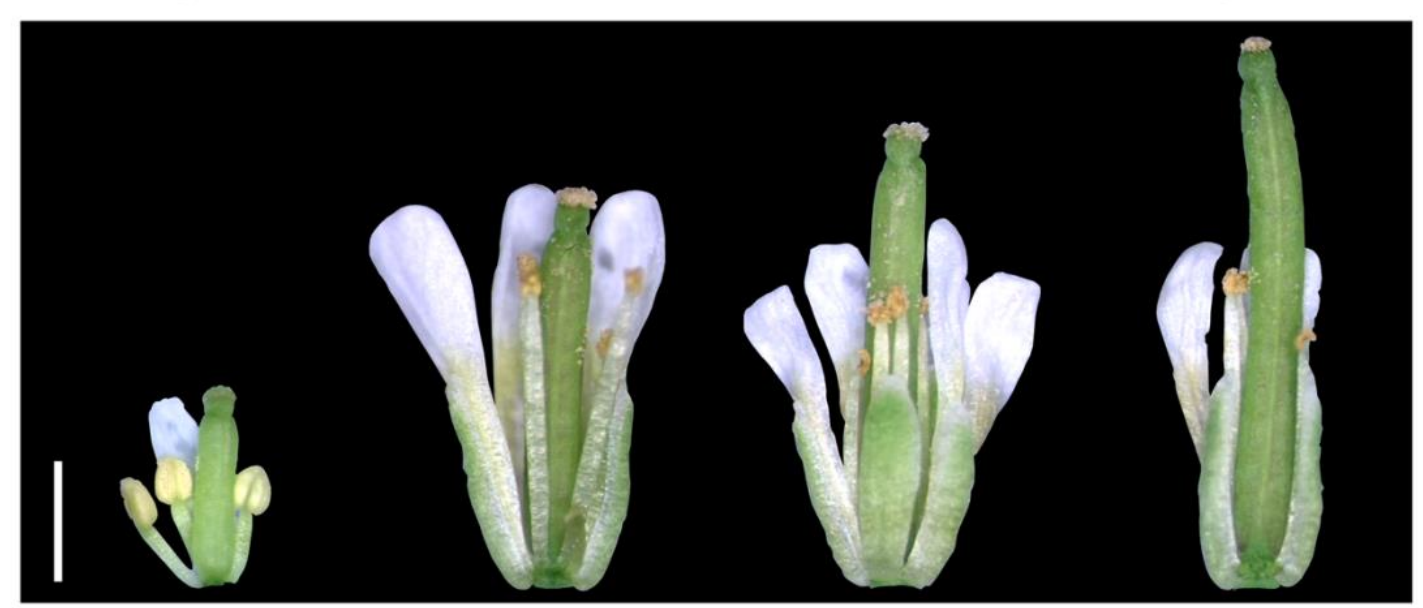

Fig. 5: AFM analysis on developing fruits.

(A) Comparison of the apparent stiffness of stk, xyll and stk/xyll mutant fruits compared to WT at different developmental stages (stage 12 before fertilization, stages 14, 15 and 16 after fertilization during fruit elongation process) which are depicted at the bottom with respect to the box plots (scale bar: $1 \mathrm{~mm}$ ). Stiffness values were extracted as Young Modulus values from the fit Hetz model of the force displacement curves in which each value represents the average Young modulus per cell square of over 100 force curves, with the associated SD. Differences between populations were evaluated statistically using a Wilcoxon rank-sum test ${ }^{*} \mathrm{p}<0.05$, $* * \mathrm{p}<0.01$ ). (B) Stereomicroscope images of WT pistil and siliques at the stages analyzed in AFM experiment; Scale bar: $1 \mathrm{~mm}$. 


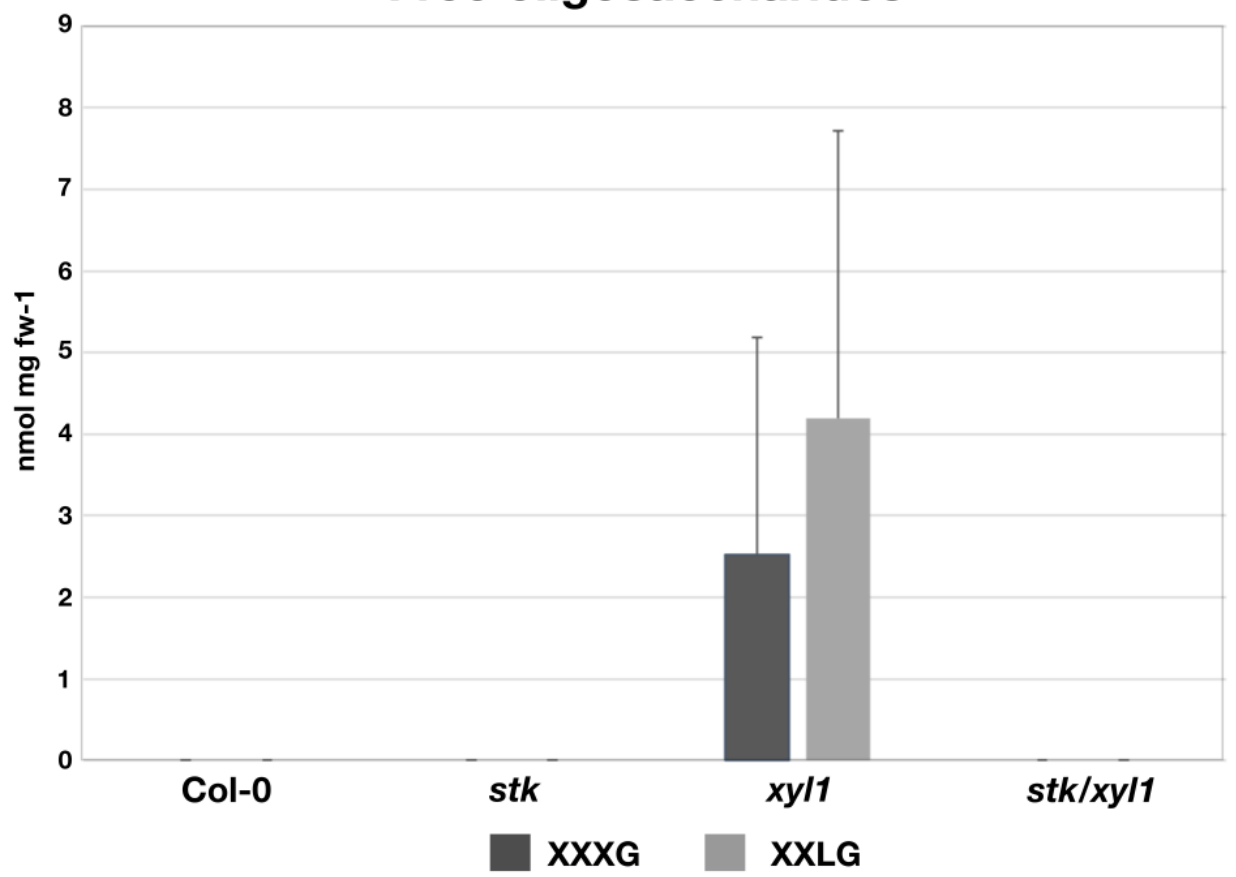

B

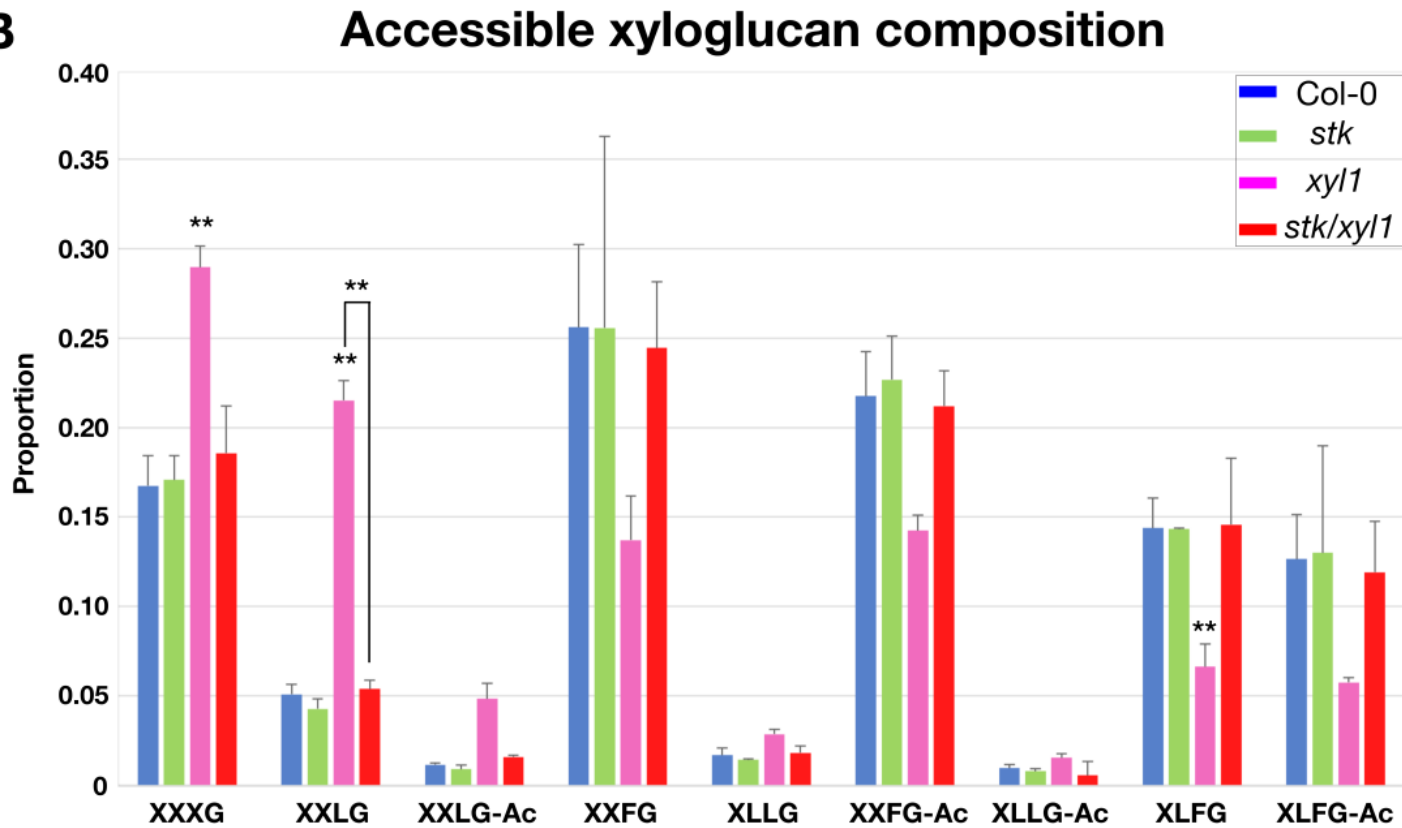

Fig. 6: Xyloglucan characterization in siliques of $s t k, x y l 1$ and $s t k / x y l 1$.

(A) Histogram representing free oligosaccharides extracted from siliques at stage 15-16 of stk, xyll and stk/xyll with respect to WT. Error bars represent the SD. $n=5$. (B) Histogram representing the proportion of accessible xyloglucan extracted from the siliques of $s t k, x y l l$ and stk/xyll with respect to WT. Error bars represent the SD. Statistical differences were identified with Student's $t$ test $(* * p<0.05)$. Mutants marked with asterisks were significantly different with respect to WT control or among them if connected. 



Fig. 7: Updated models for STK transcriptionally regulating $X Y L 1$ in the control of seed and fruit size.

(A) Proposed model for seed growth controlled by STK upstream of XYL1; STK also controls directly E2Fa to regulate seed coat cell cycle (see also (Paolo et al., 2021)). (B) Proposed model for silique growth controlled by STK upstream of XYL1. STK directly controls Cytokinin (CKs) homeostasis activating $C K X 7$ and indirectly $F U L$ which plays a crucial role in the valve elongation (see also (Di Marzo et al., 2020a)). 